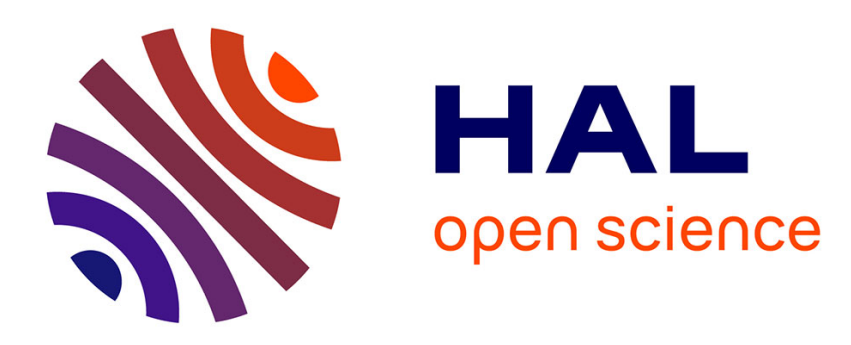

\title{
A theoretical and numerical model for the study of incompressible mixture flows
}

\author{
Franck Boyer
}

\section{To cite this version:}

Franck Boyer. A theoretical and numerical model for the study of incompressible mixture flows.

Computers and Fluids, 2002, 31 no 1, pp 41-68. hal-00004084

\section{HAL Id: hal-00004084 \\ https://hal.science/hal-00004084}

Submitted on 28 Jan 2005

HAL is a multi-disciplinary open access archive for the deposit and dissemination of scientific research documents, whether they are published or not. The documents may come from teaching and research institutions in France or abroad, or from public or private research centers.
L'archive ouverte pluridisciplinaire HAL, est destinée au dépôt et à la diffusion de documents scientifiques de niveau recherche, publiés ou non, émanant des établissements d'enseignement et de recherche français ou étrangers, des laboratoires publics ou privés. 


\title{
A theoretical and numerical model for the study of incompressible mixture flows
}

\author{
Franck Boyer ${ }^{1}$,
}

Mathématiques Appliquées de Bordeaux, Université Bordeaux 1, 351 cours de la libération, 33405 Talence cedex, France.

\begin{abstract}
In this paper we give a complete derivation of a new model for the study of incompressible mixture flows. The equations introduced are a generalization of a model previously studied in the literature, in which the densities and the viscosities of the two phases are allowed to be different.

Then, we introduce a finite-differences scheme for the numerical computations and the qualitative validation of the model. In particular, the use of an anti-diffusive second order scheme for the transport scheme is explained and justified.

One of the main physical experiment that we manage to simulate is the one of the spinodal decomposition under shear, but in order to show that the model is relevant in many general situations, we also obtain significant results in three other cases : the driven cavity, the Rayleigh-Taylor instability and the fall of a droplet.
\end{abstract}

Key Words - Navier-Stokes, Cahn-Hilliard, Order parameter, Non-homogeneous fluids, Finite differences, Spinodal decomposition.

AMS Subject Classification - 76D05, 76D45, 76D50, 76M20, 76T10.

\section{Introduction}

In this paper, a new model for the study of incompressible multiphase flows, issued from works by Onuki [23], is presented and justified. The main motivation is the study of various phenomena such as the mixing of two fluids in a driven cavity, or the spinodal decomposition under shear. The study of this last phenomenon is of great importance, as it is not yet very well understood from a physical point of view.

This phenomenon consists of a two-stages evolution of a near homogeneous initial mixture : a phase separation stage in which some macroscopic patterns appear, then a shear stage in which the patterns organize themselves into parallel layers (see [23] for experimental snapshots and the section 5 for numerical results). Hence, the model has to take into account the chemical interaction between the two phases at the interface. This is achieved using the theory of the order parameter and the associated free energy of Cahn-Hilliard type (see [16]). In this diffuse-interface theory, the thickness of the interface is not supposed to be zero unlike in the level set approaches in which the interface is flat. Nevertheless, for numerical purposes, the level set approach has to introduce an artificial thickness of the interface. The model proposed here does not need any artificial treatment of the interface, it takes naturally into account its physical thickness. As it will be shown in section 5 , the model is also able to account the changes in topology of the mixture.

Moreover, to model the hydrodynamic properties of the mixture (in the shear case for example), an equation on a mean velocity field is needed. The equation obtained is essentially a non-homogeneous Navier-Stokes equation with a surface tension term acting at the interface. This term naturally appears in the derivation as a consequence of the Cahn-Hilliard theory for the free energy of the mixture. Furthermore, the two phases are allowed to have different viscosity (for example a polymer and a solvant).

For a summary of the different kinds of models existing for these problems, see for example [2] and [18].

In the homogeneous case (when the densities of the phases are the same), such a model was already written ([10], [17]) and was studied mathematically ([5]). The mathematical study of the non homogeneous model obtained here is performed in [6].

The derivation of the model is the object of the first part of this paper (section 2). In a second part, a finite-difference numerical scheme is implemented for the discretization of the equations. More precisely, a

1e-mail: fboyer@math.u-bordeaux.fr 
scheme as simple as possible is built which allows us to observe good qualitative results. It is shown that for simple geometries with regular meshes, the finite-difference method gives quite relevant results. The main problem is the discretization of the convection term in the equations. It must be performed very carefully to follow correctly the interface, avoiding that a too high numerical diffusion induces noisy results.

Many of the existing models in the literature, such as the free boundaries formulations and the level set methods, are not so easy to use from a numerical point of view. Even the non-homogeneous fluids model ([27], [20]) which is simpler, since it does not model chemical phenomena at the interface, is in fact much more fastidious to discretize (see the case $\mathcal{P} e=+\infty$ in section 5 ).

\section{Derivation of the governing equations}

\subsection{Notations}

Consider a mixture composed of two incompressible fluids (or phases) of mass densities $\rho_{1}^{0}$ and $\rho_{2}^{0}$. These are the physical constants in the the experimental conditions. The temperature is supposed to be constant. Define the volume fraction of the first fluid in the mixture as

$$
\psi=\frac{d V_{1}}{d V}
$$

where $d V_{1}$ is the volume filled by the fluid 1 . Some authors ([13], [24]) use $\psi$ as the order parameter, in this paper a renormalized order parameter (see for example [5], [10], [14]), defined by

$$
\varphi=2 \psi-1
$$

is used so that $-1 \leq \varphi \leq 1$ and $\varphi(x)=1$ (resp. $\varphi(x)=-1$ ) if and only if the fluid 1 (resp. the fluid 2) is present at the point $x$.

The densities of each fluid in the mixture are denoted by $\rho_{1}$ and $\rho_{2}$, and $\rho$ is the total density of the mixture, defined by

$$
\begin{aligned}
\rho_{1} & =\rho_{1}^{0} \frac{1+\varphi}{2}, \\
\rho_{2} & =\rho_{2}^{0} \frac{1-\varphi}{2}, \\
\rho & =\rho_{1}+\rho_{2} .
\end{aligned}
$$

Each fluid possesses its own velocity field $\mathbf{v}_{j}$. The mean velocity $\mathbf{v}$ and the perturbation velocity $\mathbf{w}$ are defined by

$$
\begin{gathered}
\mathbf{v}=\frac{1+\varphi}{2} \mathbf{v}_{1}+\frac{1-\varphi}{2} \mathbf{v}_{2} \\
\mathbf{w}=\mathbf{v}_{1}-\mathbf{v}_{2} .
\end{gathered}
$$

\subsection{General equations}

For any vector field $\mathbf{v}$, the deformation tensor $\left(\nabla \mathbf{v}+\nabla \mathbf{v}^{t}\right) / 2$ is denoted by $\mathbf{D}(\mathbf{v})$.

- Step 1: The mass conservation of each phase reads

$$
\begin{aligned}
& \frac{\partial \rho_{1}}{\partial t}+\operatorname{div}\left(\rho_{1} \mathbf{v}_{1}\right)=0, \\
& \frac{\partial \rho_{2}}{\partial t}+\operatorname{div}\left(\rho_{2} \mathbf{v}_{2}\right)=0 .
\end{aligned}
$$

Dividing each of the equations by $\rho_{i}^{0}$, adding the equations obtained and using the definitions of $\rho_{1}$ and $\rho_{2}$, the mean velocity field is found to satisfy

$$
\operatorname{div}(\mathbf{v})=0 .
$$


Then, any of the previous conservation equations can be written as

$$
\frac{\partial \varphi}{\partial t}+\mathbf{v} \cdot \nabla \varphi+\operatorname{div}\left(\frac{1-\varphi^{2}}{2} \mathbf{w}\right)=0 .
$$

In contrast to the models of non-homogeneous fluids studied in [20] or [27], one may notice that $\rho$ does not satisfy a classical mass-conservation equation but fulfills

$$
\frac{\partial \rho}{\partial t}+\operatorname{div}(\rho \mathbf{v})+\frac{\rho_{1}^{0}-\rho_{2}^{0}}{4} \operatorname{div}\left(\left(1-\varphi^{2}\right) \mathbf{w}\right)=0 .
$$

Note also that some authors consider a massic-mean velocity defined by

$$
\rho \mathbf{v}^{*}=\rho_{1} \mathbf{v}_{1}+\rho_{2} \mathbf{v}_{2},
$$

but this velocity field is not divergence-free so that this choice is not used here.

- Step 2: The momentum conservation for each fluid reads

$$
\begin{aligned}
& \frac{\partial \rho_{1} \mathbf{v}_{1}}{\partial t}+\operatorname{div}\left(\rho_{1} \mathbf{v}_{1} \otimes \mathbf{v}_{1}\right)-\operatorname{div}\left(\boldsymbol{\sigma}_{1}\right)=\mathbf{F}_{1}, \\
& \frac{\partial \rho_{2} \mathbf{v}_{2}}{\partial t}+\operatorname{div}\left(\rho_{2} \mathbf{v}_{2} \otimes \mathbf{v}_{2}\right)-\operatorname{div}\left(\boldsymbol{\sigma}_{2}\right)=\mathbf{F}_{2},
\end{aligned}
$$

where $\sigma_{1}$ and $\sigma_{2}$ are the stress tensors of the fluids in the alloy and $F_{1}, F_{2}$ the exterior volumic forces applied on each fluid. A constitutive law is needed for these tensors and forces. The definition of the stress tensors is quite natural

$$
\begin{aligned}
& \boldsymbol{\sigma}_{1}=2 \eta_{1}(\varphi) \mathbf{D}\left(\mathbf{v}_{1}\right)-p_{1} \mathrm{Id}, \\
& \boldsymbol{\sigma}_{2}=2 \eta_{2}(\varphi) \mathbf{D}\left(\mathbf{v}_{2}\right)-p_{2} \mathrm{Id},
\end{aligned}
$$

where $p_{i}$ denotes the partial pressure, and $\eta_{i}$ the viscosity of the fluid $i$ in the mixture, which is supposed to depend on the local composition of the mixture. These viscosities are assumed to satisfy

$$
\eta_{1}(\varphi)=\frac{1+\varphi}{2} \eta(\varphi), \quad \eta_{2}(\varphi)=\frac{1-\varphi}{2} \eta(\varphi),
$$

where $\eta(\varphi)$ is the viscosity of the theoretical uniform alloy of composition $\varphi$. Moreover, we assume that the partial pressures are given from the total pressure by

$$
p_{1}=\frac{1+\varphi}{2} p, \quad p_{2}=\frac{1-\varphi}{2} p .
$$

Finally, the forces are assumed to be of the following form :

$$
\mathbf{F}_{1}=-\rho_{1} \nabla \mu_{1}+\rho_{1} \mathbf{g}-\xi(\varphi) \mathbf{w}, \quad \mathbf{F}_{2}=-\rho_{2} \nabla \mu_{2}+\rho_{2} \mathbf{g}+\xi(\varphi) \mathbf{w}
$$

where $\mathbf{g}$ is an external volumic force (gravity forces for example) and $\xi(\varphi)$ a friction coefficient depending on the composition of the alloy. The chemical potentials $\mu_{1}$ and $\mu_{2}$ of the two species are given by (cf. [23] p. 6138 and p.6146)

$$
\mu_{1}=\mu_{0}(\varphi)+\frac{1-\varphi}{2 \rho}\left(\frac{\partial \mathcal{F}}{\partial \varphi}\right), \quad \mu_{2}=\mu_{0}(\varphi)-\frac{1+\varphi}{2 \rho}\left(\frac{\partial \mathcal{F}}{\partial \varphi}\right)
$$

Here $\mu_{0}(\varphi)$ is the chemical potential of the theoretical uniform alloy of composition $\varphi$ and the other terms are exchange terms due to the non-uniformity of the alloy; $\mathcal{F}$ is the Gibbs free energy ([10],[16],[23])

$$
\mathcal{F}(\varphi)=\int_{\Omega} \frac{A}{2}|\nabla \varphi|^{2}+E F(\varphi)
$$


so that

$$
\left(\frac{\partial \mathcal{F}}{\partial \varphi}\right)=-A \Delta \varphi+E F^{\prime}(\varphi)
$$

$A$ and $E$ being two physical parameters describing the interaction between the two phases.

One of the physically-relevant expressions for the Cahn-Hilliard potential $F$ is given by a logarithmic expression ([3], [12], or [13]),

$$
F(\varphi)=\theta_{c}\left(1-\varphi^{2}\right)+\theta((1+\varphi) \log (1+\varphi)+(1-\varphi) \log (1-\varphi)),
$$

where $\theta$ is the temperature and $\theta_{c}$ the critical temperature of the mixture. In order to observe a phase separation phenomenon the temperatures are assumed to verify $\theta<\theta_{c}$ so that $F$ is not convex. For $\theta$ close enough to $\theta_{c}$, one can replace this expression by a polynomial approximation of the form

$$
F(\varphi)=C_{1} \varphi^{4}-C_{2} \varphi^{2}
$$

This polynomial form of the chemical potential is classically used in the literature ([29]).

- Step 3: Notation : For any two vectors $\mathbf{u}_{1}$ and $\mathbf{u}_{2}$, the tensor product of $\mathbf{u}_{1}$ and $\mathbf{u}_{2}$ is denoted by $\mathbf{u}_{1} \otimes \mathbf{u}_{2}$.

After some long, but straightforward computations, the equations satisfied by $\mathbf{v}$ and $\mathbf{w}$ are deduced from $(2.3)-(2.4)$ :

$$
\begin{aligned}
& \frac{\partial}{\partial t}\left(\rho \mathbf{v}+\left(\rho_{1}^{0}-\rho_{2}^{0}\right) \frac{1-\varphi^{2}}{4} \mathbf{w}\right)+\operatorname{div}\left(\rho \mathbf{v} \otimes \mathbf{v}+\frac{1-\varphi^{2}}{4}\left(\rho_{1}^{0}+\rho_{2}^{0}-\rho\right) \mathbf{w} \otimes \mathbf{w}\right) \\
&+\operatorname{div}\left(\left(\rho_{1}^{0}-\rho_{2}^{0}\right) \frac{1-\varphi^{2}}{4}(\mathbf{v} \otimes \mathbf{w}+\mathbf{w} \otimes \mathbf{v})\right) \\
&=\operatorname{div}\left(\boldsymbol{\Sigma}_{1}\right)-\operatorname{div}\left(\boldsymbol{\Sigma}_{2}\right) \\
&-\rho \nabla \mu_{0}+\left(\frac{\partial \mathcal{F}}{\partial \varphi}\right) \nabla \varphi+\rho \mathbf{g}+\left(\rho_{2}^{0}-\rho_{1}^{0}\right) \frac{1-\varphi^{2}}{4} \nabla\left(\frac{1}{\rho} \frac{\partial \mathcal{F}}{\partial \varphi}\right) . \\
& \rho_{1}^{0} \rho_{2}^{0} \frac{1-\varphi^{2}}{4} \frac{\partial \mathbf{w}}{\partial t}+\rho_{1}^{0} \rho_{2}^{0} \frac{1-\varphi^{2}}{4}(-\varphi \mathbf{w} \cdot \nabla \mathbf{w}-(\mathbf{w} \cdot \nabla \varphi) \mathbf{w}+\mathbf{v} \cdot \nabla \mathbf{w}+\mathbf{w} \cdot \nabla \mathbf{v}) \\
&=-\left(\rho_{1}^{0}-\rho_{2}^{0}\right) \operatorname{div}\left(\frac{1-\varphi^{2}}{4}\left(\boldsymbol{\Sigma}_{1}-\boldsymbol{\Sigma}_{2}\right)\right)+\rho \operatorname{div}\left(\frac{1-\varphi^{2}}{4} \boldsymbol{\Sigma}_{3}\right) \\
&+\left(\rho_{1}^{0}+\rho_{2}^{0}-\rho\right)\left(\boldsymbol{\Sigma}_{1}-\boldsymbol{\Sigma}_{2}\right) \cdot \nabla \varphi-\rho_{1}^{0} \rho_{2}^{0} \frac{1-\varphi^{2}}{4} \nabla\left(\frac{1}{\rho} \frac{\partial \mathcal{F}}{\partial \varphi}\right)-\rho \xi(\varphi) \mathbf{w}
\end{aligned}
$$

where

$$
\boldsymbol{\Sigma}_{1}=2 \eta(\varphi) \mathbf{D}(\mathbf{v})-p \mathrm{Id},
$$

is the classical stress tensor for a homogeneous incompressible fluid, and

$$
\begin{gathered}
\boldsymbol{\Sigma}_{2}=\eta(\varphi)\left(\nabla \varphi \otimes \mathbf{w}+\mathbf{w} \otimes \nabla \varphi-\frac{2}{3}(\mathbf{w} \cdot \nabla \varphi) \mathrm{Id}\right), \\
\boldsymbol{\Sigma}_{3}=2 \eta(\varphi)\left(\mathbf{D}(\mathbf{w})-\frac{1}{3} \operatorname{div}(\mathbf{w}) \mathrm{Id}\right),
\end{gathered}
$$

are two tensors involving the perturbation velocity.

One can see that these equations enter the general framework of the mechanics of mixtures in [24]. 


\subsection{Dimensionless equations and model}

In order to make the predominant terms appear, a dimensionless analysis of the system above is now performed.

- Step 1: The characteristic size of the interface is defined through a linear analysis of the steady Cahn-Hilliard equation (see [13], [16]), from the physical coefficients $A, E$, appearing in the Cahn-Hilliard free energy (2.5)-(2.6), by

$$
l=\sqrt{A / E} .
$$

Then a reference length $L$ (the size of the domain for example) is chosen and a first dimensionless parameter is introduced

$$
\alpha=l / L .
$$

The reference density is $\bar{\rho}=\max \left(\rho_{1}^{0}, \rho_{2}^{0}\right)=\rho_{1}^{0}$ (for example) and the contrast between the two densities is described by

$$
\varepsilon=\frac{\rho_{1}^{0}-\rho_{2}^{0}}{\bar{\rho}} .
$$

From a physical point of view, see [23], the dominating terms in the equation (2.8) are supposed to be the last two terms. This leads to the following choice of a reference value for $\mathbf{w}$ :

$$
\bar{w}=\frac{E}{L \bar{\xi}},
$$

by using the fact that the reference for $\frac{\partial \mathcal{F}}{\partial \varphi}$ is the physical coefficient $E$, and by denoting by $\bar{\xi}$ the characteristic value for the coefficient of friction $\xi(\varphi)$.

- Step 2: The reference velocity $\bar{v}$ is given by the problem under study - the shear velocity imposed on the boundary for example - and so the characteristic time of the flow is

$$
T=\frac{L}{\bar{v}},
$$

and the characteristic value for $\mathbf{g}$ is the acceleration $\frac{\bar{v}}{T}$. Finally, two classical dimensionless numbers, representative of the flow, are introduced :

$$
\mathcal{R} e=\frac{\bar{\rho} \bar{v} L}{\bar{\eta}}, \quad \text { the Reynolds number, }
$$

and

$$
\mathcal{K}=\frac{E T}{L \bar{\rho} \bar{v}}, \text { the capillarity coefficient (or capillary number). }
$$

For convenience, we also consider the ratio $\beta$ between the two characteristic velocities:

$$
\beta=\frac{\bar{w}}{\bar{v}}
$$

- Step 3: In dimensionless variables, the equations obtained in the previous section can be rewritten as:

$$
\begin{gathered}
\rho(\varphi)=1+\frac{\varepsilon}{2}(\varphi-1) \\
\frac{\partial \varphi}{\partial t}+\mathbf{v} \cdot \nabla \varphi+\beta \operatorname{div}\left(\frac{1-\varphi^{2}}{2} \mathbf{w}\right)=0 \\
\operatorname{div}(\mathbf{v})=0
\end{gathered}
$$


Computers and Fluids 31 numéro 1, pp 41-68 (2002)

$$
\begin{aligned}
\frac{\partial \rho \mathbf{v}}{\partial t} & +\frac{\varepsilon \beta}{4} \frac{\partial\left(1-\varphi^{2}\right) \mathbf{w}}{\partial t}+\operatorname{div}(\rho \mathbf{v} \otimes \mathbf{v})+\beta^{2} \operatorname{div}\left((\rho-\varepsilon \varphi) \frac{1-\varphi^{2}}{4} \mathbf{w} \otimes \mathbf{w}\right) \\
& +\varepsilon \beta \operatorname{div}\left(\frac{1-\varphi^{2}}{4}(\mathbf{v} \otimes \mathbf{w}+\mathbf{w} \otimes \mathbf{v})\right) \\
& =\frac{1}{\mathcal{R} e} \operatorname{div}\left(\boldsymbol{\Sigma}_{1}\right)-\frac{\beta}{\mathcal{R} e} \operatorname{div}\left(\boldsymbol{\Sigma}_{2}\right) \\
& -\frac{T}{\bar{v}} \rho \nabla \mu_{0}+\mathcal{K} \mu \nabla \varphi+\varepsilon \mathcal{K} \frac{1-\varphi^{2}}{4} \nabla\left(\frac{\mu}{\rho}\right)+\rho \mathbf{g},
\end{aligned}
$$

with

$$
\begin{aligned}
\mu & =-\alpha^{2} \Delta \varphi+F^{\prime}(\varphi) \\
(1-\varepsilon) \frac{\bar{\rho}}{T \bar{\xi}} \frac{1-\varphi^{2}}{4} \frac{\partial \mathbf{w}}{\partial t} & -(1-\varepsilon) \frac{\overline{\mathbf{w}} \bar{\rho}}{L \bar{\xi}} \frac{1-\varphi^{2}}{4}(\varphi \mathbf{w} \cdot \nabla \mathbf{w}+(\mathbf{w} \cdot \nabla \varphi) \mathbf{w})+(1-\varepsilon) \frac{\bar{\rho} \bar{v}}{L \bar{\xi}} \frac{1-\varphi^{2}}{4}(\mathbf{v} \cdot \nabla \mathbf{w}+\mathbf{w} \cdot \nabla \mathbf{v}) \\
& =-\frac{\varepsilon}{\mathcal{R} e} \frac{\bar{\rho} \bar{v}^{2}}{L \bar{\xi} \bar{w}} \operatorname{div}\left(\frac{1-\varphi^{2}}{4} \boldsymbol{\Sigma}_{1}\right)+\frac{\varepsilon}{\mathcal{R} e} \frac{\bar{\rho} \bar{v}}{L \bar{\xi}} \operatorname{div}\left(\frac{1-\varphi^{2}}{4} \boldsymbol{\Sigma}_{2}\right) \\
& +\frac{1}{\mathcal{R} e} \frac{\bar{\rho} \bar{v}}{L \bar{\xi}} \rho \operatorname{div}\left(\frac{1-\varphi^{2}}{4} \boldsymbol{\Sigma}_{3}\right)+\frac{1}{\mathcal{R} e} \frac{\bar{\rho} \bar{v}^{2}}{L \bar{\xi} \bar{w}}(\rho-\varepsilon \varphi) \boldsymbol{\Sigma}_{1} \cdot \nabla \varphi \\
& -\frac{1}{\mathcal{R} e} \frac{\bar{\rho} \bar{v}}{L \bar{\xi}}(\rho-\varepsilon \varphi) \boldsymbol{\Sigma}_{2} \cdot \nabla \varphi \\
& -(1-\varepsilon) \frac{1-\varphi^{2}}{4} \nabla\left(\frac{\mu}{\rho}\right)-\rho \xi(\varphi) \mathbf{w} .
\end{aligned}
$$

- Step 4: Some particular values of the physical parameters corresponding to a mixture of a polymer and water, at a temperature $\theta$ such that $\theta-\theta_{c} \sim 1 \mathrm{~K}$ ([11], [23]), are now used :

$$
\bar{\eta}=10^{-3} \text { Pa.s }, \quad \bar{\rho}=10^{3} \mathrm{~kg} \cdot \mathrm{m}^{-3}, \quad E=10^{7} \mathrm{~Pa}, \quad \bar{\xi}=10^{15} \mathrm{~kg} \cdot \mathrm{m}^{-3} \cdot \mathrm{s}^{-1}, \quad l=10^{-9} \mathrm{~m} .
$$

If the mixture is under shear between two cylinders, one can take in classical experimental conditions

$$
L=10^{-3} \mathrm{~m}, \quad \bar{v}=10^{-1} \mathrm{~m} \cdot \mathrm{s}^{-1},
$$

which gives the following values for the other parameters :

$$
T=10^{-2} \mathrm{~s}, \quad \alpha=10^{-6}, \quad \bar{w}=10^{-5} \mathrm{~m} . \mathrm{s}^{-1}, \quad \beta=\frac{\bar{w}}{\bar{v}}=10^{-4}, \quad \mathcal{K}=10^{6}, \quad \mathcal{R} e=100 .
$$

Finally, one can deduce that the equation (2.17) can be approached by the equation

$$
\xi(\varphi) \mathbf{w}=-\frac{\left(1-\varphi^{2}\right)(1-\varepsilon)}{4 \rho} \nabla\left(\frac{\mu}{\rho}\right)
$$

and the equation (2.15), by

$$
\begin{aligned}
\frac{\partial \rho \mathbf{v}}{\partial t}+\operatorname{div}(\rho \mathbf{v} \otimes \mathbf{v}) & -\frac{2}{\mathcal{R} e} \operatorname{div}(\eta(\varphi) \mathbf{D}(\mathbf{v}))+\nabla p \\
& =\mathcal{K} \mu \nabla \varphi+\varepsilon \mathcal{K} \frac{1-\varphi^{2}}{4} \nabla\left(\frac{\mu}{\rho}\right)+\rho \mathbf{g} .
\end{aligned}
$$

Note that the pressure gradient term appears from the definition (2.9) of $\Sigma_{1}$. 
This last equation is put in a non-conservative form (which is useful for the numerical study of the problem) to obtain

$$
\begin{aligned}
\rho\left(\frac{\partial \mathbf{v}}{\partial t}+\mathbf{v} \cdot \nabla \mathbf{v}\right) & -\frac{2}{\mathcal{R} e} \operatorname{div}(\eta(\varphi) \mathbf{D}(\mathbf{v}))+\nabla p \\
& =\mathcal{K} \mu \nabla \varphi+\varepsilon \mathcal{K} \frac{1-\varphi^{2}}{4} \nabla\left(\frac{\mu}{\rho}\right)+\rho \mathbf{g} \\
& -\varepsilon(1-\varepsilon) \alpha \beta \operatorname{div}\left(\frac{\left(1-\varphi^{2}\right)^{2}}{8 \rho \xi(\varphi)} \nabla\left(\frac{\mu}{\rho}\right)\right) \cdot \mathbf{v}
\end{aligned}
$$

The last term above is negligible compared to the others so that, the equation considered further is the following:

$$
\begin{aligned}
\rho\left(\frac{\partial \mathbf{v}}{\partial t}+\mathbf{v} \cdot \nabla \mathbf{v}\right) & -\frac{2}{\mathcal{R} e} \operatorname{div}(\eta(\varphi) \mathbf{D}(\mathbf{v}))+\nabla p \\
& =\mathcal{K} \mu \nabla \varphi+\varepsilon \mathcal{K} \frac{1-\varphi^{2}}{4} \nabla\left(\frac{\mu}{\rho}\right)+\rho \mathbf{g}
\end{aligned}
$$

\section{$2.4 \quad$ Final model}

Under the previous approximations, the system of equations can be written as:

$$
\begin{aligned}
& \rho(\varphi)=1+\frac{\varepsilon}{2}(\varphi-1), \\
& \frac{\partial \varphi}{\partial t}+\mathbf{v} \cdot \nabla \varphi-\frac{1}{\mathcal{P} e} \operatorname{div}\left(\frac{B(\varphi)}{\rho(\varphi)} \nabla\left(\frac{\mu}{\rho}\right)\right)=0, \\
& \mu=-\alpha^{2} \Delta \varphi+F^{\prime}(\varphi), \\
& \rho\left(\frac{\partial \mathbf{v}}{\partial t}+\mathbf{v} \cdot \nabla \mathbf{v}\right)-\frac{1}{\mathcal{R} e} \operatorname{div}(2 \eta(\varphi) \mathbf{D}(\mathbf{v}))+\nabla p=\mathcal{K} \mu \nabla \varphi+\varepsilon \mathcal{K} \frac{1-\varphi^{2}}{4} \nabla\left(\frac{\mu}{\rho}\right)+\rho \mathbf{g}, \\
& \operatorname{div} \mathbf{v}=0,
\end{aligned}
$$

where the following dimensionless numbers are used

$$
B(\varphi)=\frac{\left(1-\varphi^{2}\right)^{2}}{8 \xi(\varphi)} \quad(\text { mobility coefficient }), \quad \mathcal{P} e=\frac{1}{(1-\varepsilon) \beta} \quad \text { (Peclet number) }
$$

One can easily recognize in this system a generalization of the system studied in [5], [10] when the two fluids have same densities $(\varepsilon=0, \rho(\varphi) \equiv 1)$ and which was derived by different methods in [18], [17], [19]. Of course our model can be also used, for other values of the physical parameters that ones considered here.

One may notice that $\rho$ satisfies the equation

$$
\frac{\partial \rho}{\partial t}+\mathbf{v} \cdot \nabla \rho-\frac{\varepsilon}{2 \mathcal{P} e} \operatorname{div}\left(\frac{B(\varphi)}{\rho(\varphi)} \nabla\left(\frac{\mu}{\rho}\right)\right)=0 .
$$

In the next sections, the numerical approximation of this system of equations in $2 \mathrm{D}$ is investigated. In particular, two cases of numerical experiments are considered : the mixing of two fluids in a driven cavity 
which can be compared to previous works [10], and the spinodal decomposition under shear in a channel which is of a great physical interest (see [23]).

It will be also shown that the present model can be used for studying, for example, a well-known case of instability - the Rayleigh-Taylor instability - or the fall of a droplet of water (resp. of mercury) in the air (resp. in the water).

In all cases, the computational domain is rectangular, the only difference being in the choice of the boundary conditions. In the case of the driven cavity, the velocity is imposed on the boundary and satisfies $\mathbf{v} . \boldsymbol{\nu}=0$, whereas the boundary conditions for $\varphi$ are the natural no-flux conditions

$$
\frac{\partial \varphi}{\partial \nu}=\frac{\partial \mu}{\partial \nu}=0
$$

the first condition imposing locally the interface to be orthogonal to the walls. In the case of the channel under shear, periodicity conditions are imposed for $\varphi, \mu$ and $\mathbf{v}$ in the longitudinal $x$ direction. The periodicity conditions are natural because in the physical experiments the shear is obtained by putting the mixture between two rotating cylinders whose diameters are very close (Couette-Taylor flow), in which curvature effects can be neglected because of the thickness of the domain.

The time-discretization chosen for this system is presented in section 3, then the finite-differences schemes used for the space-discretization are explained in section 4. Finally, the results are presented and commented for each test case (section 5).

\section{Time discretization}

From now on, the two coordinates of the velocity $\mathbf{v}$ will be denoted by

$$
\mathbf{v}=\left(\begin{array}{l}
u \\
v
\end{array}\right)
$$

The time-discretization is carried on, with a time step $\delta t$, through a three steps finite difference scheme as follows.

- Step 1 - Cahn-Hilliard equation

Provided approximations $\varphi^{n}, \mu^{n}$ and $\mathbf{v}^{n}$ of the solution at time $t_{n}$ are known, the resolution of the CahnHilliard part of the system is first performed, namely $\varphi^{n+1 / 2}, \mu^{n+1 / 2}$ are computed as solutions of

$$
\begin{array}{r}
\frac{\varphi^{n+1 / 2}-\varphi^{n}}{\delta t}-\frac{1}{\mathcal{P} e} \operatorname{div}\left(\frac{B\left(\varphi^{n}\right)}{\rho\left(\varphi^{n}\right)} \nabla\left(\frac{\theta \mu^{n+1 / 2}+(1-\theta) \mu^{n}}{\rho\left(\varphi^{n}\right)}\right)\right)=0, \\
\left(\theta \mu^{n+1 / 2}+(1-\theta) \mu^{n}\right)+\alpha^{2} \Delta\left(\theta \varphi^{n+1 / 2}+(1-\theta) \varphi^{n}\right)=F^{\prime}\left(\theta \varphi^{n+1 / 2}+(1-\theta) \varphi^{n}\right) .
\end{array}
$$

A $\theta$-scheme is used, which is well-known to be unconditionally stable for $\frac{1}{2} \leq \theta \leq 1$, and of order 2 if $\theta=\frac{1}{2}$. In order to avoid the limit cases of stability the value of $\theta$ is chosen slightly higher than $\frac{1}{2}$. The computations presented in section 5 were performed with $\theta=0.6$.

In order to solve (3.1)-(3.2), a fixed-point method is used. More precisely, setting $\Phi^{n+1 / 2,0}=\varphi^{n}$ and $\mathcal{M}^{n+1 / 2,0}=\mu^{n}, \Phi^{n+1 / 2, k}$ and $\mathcal{M}^{n+1 / 2, k}$ are successively obtained from the linear system

$$
\begin{array}{r}
\Phi^{n+1 / 2, k+1}-\frac{\theta \delta t}{\mathcal{P} e} \operatorname{div}\left(\frac{B\left(\varphi^{n}\right)}{\rho\left(\varphi^{n}\right)} \nabla\left(\frac{\mathcal{M}^{n+1 / 2, k+1}}{\rho\left(\varphi^{n}\right)}\right)\right)=\varphi^{n} \\
\mathcal{M}^{n+1 / 2, k+1}+\alpha^{2} \Delta \Phi^{n+1 / 2, k+1}=F^{\prime}\left(\Phi^{n+1 / 2, k}\right) .
\end{array}
$$

The sequences $\left(\Phi^{n+1 / 2, k}\right)_{k}$ and $\left(\mathcal{M}^{n+1 / 2, k}\right)_{k}$ converge respectively to $\theta \varphi^{n+1 / 2}+(1-\theta) \varphi^{n}$, and $\theta \mu^{n+1 / 2}+(1-\theta) \mu^{n}$ from which the values of $\varphi^{n+1 / 2}$ and $\mu^{n+1 / 2}$ are deduced. In practice only a few iterations in $k$ are necessary 
to reach the convergence. In some rare cases in which the rate of convergence of the fixed-point method is not sufficient, a Newton algorithm has to be used to solve these non-linear equations more efficiently.

- Step 2 - Transport term

Here, the convection term in (2.21), which is not taken into account in the previous step, is discretized. One of the two following explicit schemes is used:

- First order scheme with a Murman scheme for spatial discretization:

$$
\varphi^{n+1}=\varphi^{n+1 / 2}-\delta t \mathbf{v}^{n} \cdot \nabla \varphi^{n+1 / 2},
$$

- Second order scheme with a stabilized, anti-diffusive correction of the scheme: (see [7], [8], [25] and section 4)

$$
\begin{gathered}
\varphi^{n+1}=\varphi^{n+1 / 2}-\delta t \mathbf{v}^{n} \cdot \nabla \varphi^{n+1 / 2}+\frac{\delta t^{2}}{2} \operatorname{div}\left(\mathbf{D}^{*}\left(\mathbf{v}^{n}\right) \cdot \nabla \varphi^{n+\frac{1}{2}}\right), \\
\mathbf{D}^{*}(\mathbf{v})=\left(\begin{array}{cc}
u^{2} & u v \\
u v & v^{2}
\end{array}\right) .
\end{gathered}
$$

In the two cases the values of $\mu^{n+1}$ are computed from the values of $\varphi^{n+1}$ in a straightforward way

$$
\mu^{n+1}=-\alpha^{2} \Delta \varphi^{n+1}+F^{\prime}\left(\varphi^{n+1}\right) .
$$

As usual we will see later that this part of the scheme is the only one (with the other transport term $\mathbf{v} . \nabla \mathbf{v}$ in the Navier-Stokes equation) which introduces a stability condition.

- Step 3 - Navier-Stokes equation

For the discretization of the Navier-Stokes equation, the method used is implicit for the pressure and for the viscous terms and explicit for the inertial term and the coupling term. More precisely, $\varphi^{n+1}, \mu^{n+1}$ and $\mathbf{v}^{n}$ given, $\mathbf{v}^{n+1}$ and $p^{n+1}$ are computed from

$$
\begin{aligned}
\rho\left(\varphi^{n+1}\right)\left(\frac{\mathbf{v}^{n+1}-\mathbf{v}^{n}}{\delta t}\right)- & \frac{1}{\mathcal{R} e} \operatorname{div}\left(2 \eta\left(\varphi^{n+1}\right) \mathbf{D}\left(\mathbf{v}^{n+1}\right)\right)+\nabla p^{n+1} \\
= & -\rho\left(\varphi^{n+1}\right) \mathbf{v}^{n} \cdot \nabla \mathbf{v}^{n}+\mathcal{K} \mu^{n+1} \nabla \varphi^{n+1} \\
+ & \varepsilon \mathcal{K} \frac{1-\left(\varphi^{n+1}\right)^{2}}{4} \nabla\left(\frac{\mu^{n+1}}{\rho\left(\varphi^{n+1}\right)}\right)+\rho\left(\varphi^{n+1}\right) \mathbf{g}, \\
& \operatorname{div}\left(\mathbf{v}^{n+1}\right)=0 .
\end{aligned}
$$

Recall that $\rho\left(\varphi^{n+1}\right)$ is directly obtained from $\varphi^{n+1}$ by $(2.20)$.

Finally, solving a general Stokes problem is needed. This is made by an augmented lagrangian method [15]: given $r>0$ large enough (in the computations presented in section 5 , the values of $r$ used are in $\left[\frac{10^{2}}{\mathcal{R} e}, \frac{10^{5}}{\mathcal{R} e}\right]$ ), letting $p^{n+1,0}=p^{n}$ and $\mathbf{v}^{n+1,0}=\mathbf{v}^{n}$, the following elliptic linear problem is solved

$$
\begin{aligned}
\rho\left(\varphi^{n+1}\right)\left(\frac{\mathbf{v}^{n+1, k+1}-\mathbf{v}^{n}}{\delta t}\right) & -\frac{1}{\mathcal{R} e} \operatorname{div}\left(2 \eta\left(\varphi^{n+1}\right) \mathbf{D}\left(\mathbf{v}^{n+1, k+1}\right)\right)-r \nabla \operatorname{div} \mathbf{v}^{n+1, k+1} \\
& =-\nabla p^{n+1, k}-\rho\left(\varphi^{n+1}\right) \mathbf{v}^{n} \cdot \nabla \mathbf{v}^{n} \\
& +\mathcal{K} \mu^{n+1} \nabla \varphi^{n+1}+\varepsilon \mathcal{K} \frac{1-\left(\varphi^{n+1}\right)^{2}}{4} \nabla\left(\frac{\mu^{n+1}}{\rho\left(\varphi^{n+1}\right)}\right)+\rho\left(\varphi^{n+1}\right) \mathbf{g} .
\end{aligned}
$$

The term $p^{n+1, k+1}$ is deduced through

$$
p^{n+1, k+1}-p^{n+1, k}+r \operatorname{div} \mathbf{v}^{n+1, k+1}=0 .
$$

The iterations in $k$ are made until a small enough divergence for $\mathbf{v}^{n+1, k}$ (in the $L^{2}$-norm for example) is reached. In fact, a few number of iterations in $k$ are necessary. The velocity field $\mathbf{v}^{n+1}$ and the pressure field $p^{n+1}$ found after convergence are clearly approximated solutions of (3.8). 


\section{Space discretization and effective resolution}

\subsection{Description of the mesh and the discrete unknowns}

We suppose the domain to be rectangular, in order to be able to treat the case of a mixture in a cavity (with Dirichlet boundary conditions), but also the case of a mixture in a channel under shear (with periodicity conditions). Moreover, mesh is chosen regular, composed of $N \times M$ rectangular and isometric cells of size $\delta x \times \delta y$. The points where the solutions of the equations are sought, are chosen in a classical way as indicated in figure 1 and are denoted by $A_{i, j}, A_{i \pm \frac{1}{2}, j}$, and $A_{i, j \pm \frac{1}{2}}$.

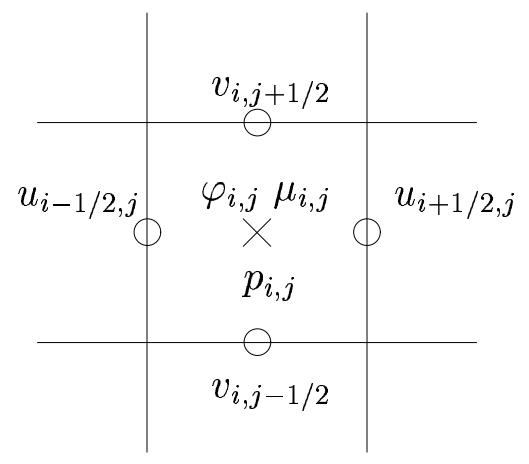

Figure 1: Location of the unknowns for the $(i, j)$-cell $C_{i, j}$

\subsection{Treatment of the boundary conditions}

Taking into account the boundary conditions classically requires the introduction of artificial discrete unknowns. For example, as far as the order parameter $\varphi$ is concerned we compute also $\varphi_{i, j}$ for $i=0, N+1$, $j=1, \ldots, M$, and for $j=0, M+1, i=0, \ldots, N+1$. Then the relations satisfied by the artificial unknowns are derived from the boundary conditions. In the example of the order parameter, the equations are the following:

- Case of the cavity : The boundary condition $\frac{\partial \varphi}{\partial \boldsymbol{\nu}}=0$ on $\partial \Omega$ gives

$$
\begin{gathered}
\frac{\varphi_{0, j}-\varphi_{1, j}}{\delta x}=\frac{\varphi_{N, j}-\varphi_{N+1, j}}{\delta x}=0 \text { for } j=1, \ldots, M, \\
\frac{\varphi_{i, 0}-\varphi_{i, 1}}{\delta y}=\frac{\varphi_{i, M}-\varphi_{i, M+1}}{\delta y}=0, \text { for } i=0, \ldots, N+1 .
\end{gathered}
$$

- Case of the channel in the $x$ direction : On the upper and the lower boundaries of $\Omega$ the boundary condition is once again $\frac{\partial \varphi}{\partial \nu}=0$ so that we have

$$
\frac{\varphi_{i, 0}-\varphi_{i, 1}}{\delta y}=\frac{\varphi_{i, M}-\varphi_{i, M+1}}{\delta y}=0, \text { for } i=0, \ldots, N+1,
$$

whereas on the left and right boundaries the periodicity condition reads

$$
\varphi_{0, j}-\varphi_{N, j}=\varphi_{1, j}-\varphi_{N+1, j}=0 \text { for } j=1, \ldots, M .
$$

One can easily write similar relations for $\mu, u, v, p$ and for the intermediate quantities $\Phi, \mathcal{M}$.

This method enjoy numerous advantages : its implementation is easy and it can be easily extended to more complex kind of boundary conditions. Moreover, it allows the discretization of the equations (2.21)-(2.23) in the same way in each point in the interior of the domain, even for the points near the boundaries. 


\subsection{Discretization of the equations}

As a consequence of the previous section, it is sufficient now to discretize the equations in each point in the interior of the domain. If necessary, values of the artificial unknowns, obtained directly from the boundary conditions relations written above, are used outside the physical domain.

\subsubsection{Cahn-Hilliard equation}

In the time discretization proposed in section 3, it is necessary to solve at each time step the system (3.3)-(3.4), which can be written in a more general form in the following way

$$
\begin{aligned}
\Phi-\omega \operatorname{div}\left(\frac{\tilde{B}}{\tilde{\rho}} \nabla\left(\frac{\mathcal{M}}{\tilde{\rho}}\right)\right) & =a, \\
\mathcal{M}+\alpha^{2} \Delta \Phi & =b,
\end{aligned}
$$

where $a, b, \tilde{B}=B\left(\varphi^{n}\right)$ and $\tilde{\rho}=\rho\left(\varphi^{n}\right)$ are known at the points $A_{i, j}$. The scheme used is of second order, and is classically given in each point $A_{i, j}$ by

$$
\begin{aligned}
& \Phi_{i, j}-\omega \frac{1}{\delta x^{2}}\left(\frac{\tilde{B}_{i+\frac{1}{2}, j}}{\tilde{\rho}_{i+\frac{1}{2}, j}}\left(\frac{\mathcal{M}_{i+1, j}}{\tilde{\rho}_{i+1, j}}-\frac{\mathcal{M}_{i, j}}{\tilde{\rho}_{i, j}}\right)-\frac{\tilde{B}_{i-\frac{1}{2}, j}}{\tilde{\rho}_{i-\frac{1}{2}, j}}\left(\frac{\mathcal{M}_{i, j}}{\tilde{\rho}_{i, j}}-\frac{\mathcal{M}_{i-1, j}}{\tilde{\rho}_{i-1, j}}\right)\right) \\
&-\omega \frac{1}{\delta y^{2}}\left(\frac{\tilde{B}_{i, j+\frac{1}{2}}}{\tilde{\rho}_{i, j+\frac{1}{2}}}\left(\frac{\mathcal{M}_{i, j+1}}{\tilde{\rho}_{i, j+1}}-\frac{\mathcal{M}_{i, j}}{\tilde{\rho}_{i, j}}\right)-\frac{\tilde{B}_{i, j-\frac{1}{2}}}{\tilde{\rho}_{i, j-\frac{1}{2}}}\left(\frac{\mathcal{M}_{i, j}}{\tilde{\rho}_{i, j}}-\frac{\mathcal{M}_{i, j-1}}{\tilde{\rho}_{i, j-1}}\right)\right)=a_{i, j}
\end{aligned}
$$

and

$$
\mathcal{M}_{i, j}+\alpha^{2} \frac{\Phi_{i+1, j}-2 \Phi_{i, j}+\Phi_{i-1, j}}{\delta x^{2}}+\alpha^{2} \frac{\Phi_{i, j+1}-2 \Phi_{i, j}+\Phi_{i, j-1}}{\delta y^{2}}=b_{i, j}
$$

where $\tilde{B}_{i \pm \frac{1}{2}, j}$ stands for the harmonic mean of $\tilde{B}_{i, j}$ and $\tilde{B}_{i \pm 1, j}$, and $\tilde{B}_{i, j \pm \frac{1}{2}}$ stands for the harmonic mean of $\tilde{B}_{i, j}$ and $\tilde{B}_{i, j \pm 1}$.

Finally, a linear system for the unknowns $\left(\Phi_{i, j}\right),\left(\mathcal{M}_{i, j}\right)$ is obtained. It can be written in a simplified matricial form :

$$
\left(\begin{array}{cc}
I & -\omega \operatorname{div}\left(\frac{\tilde{B}}{\tilde{\rho}} \nabla(\dot{\tilde{\tilde{\rho}}})\right) \\
\alpha^{2} \Delta & I
\end{array}\right)\left(\begin{array}{c}
\Phi \\
\mathcal{M}
\end{array}\right)=\left(\begin{array}{c}
a \\
b
\end{array}\right) .
$$

\subsubsection{Convection term}

In section 3, two schemes for the convective term in the Cahn-Hilliard equation were proposed: (3.5) and (3.6). For each of them, a corresponding spatial discretization is now explained.

In both cases, the scheme has to be conservative and $L^{\infty}$-stable so that the values of $\varphi$ will be always meaningful (that is to say in $[-1,1]$ ).

The first one is a variant of the classical upwind scheme, which is conservative and is known as the Murman scheme. This scheme is very easy to implement and is known to be efficient in certain kind of problems. Unfortunately, in the further tests (section 5), it is shown that this scheme leads to an important numerical diffusion in our situation, which tends to make the interface more and more diffuse and finally to give some bad results for long time computations.

The second scheme presented is a centered scheme with anti-diffusive terms and flux limiters proposed in [25]. The same kind of scheme was studied in the one-dimensional case in [7], and in the finite volume case in [8]. It enjoys the different properties needed (conservativity and $L^{\infty}$-stability) and the numerical diffusion is highly limited. Obviously this second scheme is much more complicated to derive and to implement but it will be shown in section 4.4 that it gives better results for the model under study. In fact, it was shown in [7], [8], 
[25] that this scheme is one of the most accurate compared to other classical schemes for convection-diffusion problems.

In the two cases the $L^{\infty}$-stability is ensured under a CFL condition.

Notation : From now on the symbol $\Delta$ will denote the discrete difference operators. As an example, $\Delta \varphi_{i+\frac{1}{2}, j}$ stands for the difference $\varphi_{i+1, j}-\varphi_{i, j}$.

\section{- Murman scheme}

The right-hand-side of equation (3.5) is discretized in the following way :

$$
\begin{aligned}
(\mathbf{v} . \nabla \varphi)_{i, j} & =\left(u_{i-\frac{1}{2}, j}\right)^{+}\left(\frac{\Delta \varphi_{i-\frac{1}{2}, j}}{\delta x}\right)-\left(u_{i+\frac{1}{2}, j}\right)^{-}\left(\frac{\Delta \varphi_{i+\frac{1}{2}, j}}{\delta x}\right) \\
& +\left(v_{i, j-\frac{1}{2}}\right)^{+}\left(\frac{\Delta \varphi_{i, j-\frac{1}{2}}}{\delta y}\right)-\left(v_{i, j+\frac{1}{2}}\right)^{-}\left(\frac{\Delta \varphi_{i, j+\frac{1}{2}}}{\delta y}\right),
\end{aligned}
$$

where $u^{+}$and $u^{-}$denote respectively the positive and the negative part of $u\left(u=u^{+}-u^{-}\right)$.

\section{Proposition 4.1}

The Murman scheme is conservative and $L^{\infty}$-stable under the CFL condition

$$
\frac{\delta t}{\delta x} \sup _{i, j}\left|u_{i-\frac{1}{2}, j}\right|+\frac{\delta t}{\delta y} \sup _{i, j}\left|v_{i, j-\frac{1}{2}}\right| \leq 1 .
$$

\section{- Anti-diffusive scheme}

In this section, following [25], the discretization of (3.6) is presented. First the equation (3.6) is replaced by

$$
\begin{gathered}
\varphi^{n+1}=\varphi^{n+1 / 2}-\delta t \mathbf{v}^{n} \cdot \nabla \varphi^{n+1 / 2}+\frac{\delta t^{2}}{2} \operatorname{div}\left(\mathbf{D}^{* *}\left(\mathbf{v}^{n}\right) \cdot \nabla \varphi^{n+\frac{1}{2}}\right) \\
\mathbf{D}^{* *}(\mathbf{v})=\left(\begin{array}{cc}
\max \left(u^{2}, \frac{\lambda_{y}}{\lambda_{x}}|u v|\right) & u v \\
u v^{2} & \max \left(v^{2}, \frac{\lambda_{x}}{\lambda_{y}}|u v|\right)
\end{array}\right)
\end{gathered}
$$

where $\lambda_{x}=\frac{\delta t}{\delta x}$ and $\lambda_{y}=\frac{\delta t}{\delta y}$.

Note that the scheme obtained is no more of second order in time. Nevertheless, the loss of accuracy is very limited and negligible compared to the advantages of the method (namely the limitation of the numerical diffusion). Indeed, the goal of this modification is to slightly increase the natural diffusion of the equation in order to be able to prove the stability of the scheme.

Now, (4.2) is discretized by centered finite-differences, with upwind approximation of the fluxes when necessary. Finally, all the terms obtained are separated into the diffusive terms and the anti-diffusive terms to obtain, where the superscripts $n+\frac{1}{2}$ and $n$ on $\varphi^{n+\frac{1}{2}}$ and $\mathbf{v}^{n}$ are dropped for convenience reasons:

$$
\begin{aligned}
\varphi_{i, j}^{*}= & \varphi_{i, j}-\lambda_{x} \gamma_{i, j}\left(u_{i-\frac{1}{2}, j}^{+} \Delta \varphi_{i-\frac{1}{2}, j}-u_{i+\frac{1}{2}, j}^{-} \Delta \varphi_{i+\frac{1}{2}, j}\right) \\
& -\lambda_{y} \beta_{i, j}\left(v_{i, j-\frac{1}{2}}^{+} \Delta \varphi_{i, j-\frac{1}{2}}-v_{i, j+\frac{1}{2}}^{-} \Delta \varphi_{i, j+\frac{1}{2}}\right) \\
& -\frac{\lambda_{x} \lambda_{y}}{2}\left(u_{i+\frac{1}{2}, j}^{-}\left(v_{i+1, j-\frac{1}{2}}^{+} \Delta \varphi_{i+1, j-\frac{1}{2}}-v_{i+1, j+\frac{1}{2}}^{-} \Delta \varphi_{i+1, j+\frac{1}{2}}\right)\right. \\
& +u_{i-\frac{1}{2}, j}^{+}\left(v_{i-1, j-\frac{1}{2}}^{+} \Delta \varphi_{i-1, j-\frac{1}{2}}-v_{i-1, j+\frac{1}{2}}^{-} \Delta \varphi_{i-1, j+\frac{1}{2}}\right) \\
& +v_{i, j+\frac{1}{2}}^{-}\left(u_{i-\frac{1}{2}, j+1}^{+} \Delta \varphi_{i-\frac{1}{2}, j+1}-u_{i+\frac{1}{2}, j+1}^{-} \Delta \varphi_{i+\frac{1}{2}, j+1}\right) \\
& \left.+v_{i, j-\frac{1}{2}}^{+}\left(u_{i-\frac{1}{2}, j-1}^{+} \Delta \varphi_{i-\frac{1}{2}, j-1}-u_{i+\frac{1}{2}, j-1}^{-} \Delta \varphi_{i+\frac{1}{2}, j-1}\right)\right),
\end{aligned}
$$


and

$$
\begin{aligned}
\varphi_{i, j}^{n+1}= & \varphi_{i, j}^{*}-\frac{\lambda_{x}}{2}\left(Q_{i+\frac{1}{2}, j} \Delta \varphi_{i+\frac{1}{2}, j}-Q_{i-\frac{1}{2}, j} \Delta \varphi_{i-\frac{1}{2}, j}\right) \\
& -\frac{\lambda_{y}}{2}\left(R_{i, j+\frac{1}{2}} \Delta \varphi_{i, j+\frac{1}{2}}-R_{i, j-\frac{1}{2}} \Delta \varphi_{i, j-\frac{1}{2}}\right) \\
& -\frac{\lambda_{x} \lambda_{y}}{4}\left(u_{i+\frac{1}{2}, j}^{-}\left(\left|v_{i+1, j+\frac{1}{2}}\right| \Delta \varphi_{i+1, j+\frac{1}{2}}-\left|v_{i+1, j-\frac{1}{2}}\right| \Delta \varphi_{i+1, j-\frac{1}{2}}\right)\right. \\
& +u_{i-\frac{1}{2}, j}^{+}\left(\left|v_{i-1, j+\frac{1}{2}}\right| \Delta \varphi_{i-1, j+\frac{1}{2}}-\left|v_{i-1, j-\frac{1}{2}}\right| \Delta \varphi_{i-1, j-\frac{1}{2}}\right) \\
& +v_{i, j+\frac{1}{2}}^{-}\left(\left|u_{i+\frac{1}{2}, j+1}\right| \Delta \varphi_{i+\frac{1}{2}, j+1}-\left|u_{i-\frac{1}{2}, j+1}\right| \Delta \varphi_{i-\frac{1}{2}, j+1}\right) \\
& \left.+v_{i, j-\frac{1}{2}}^{+}\left(\left|u_{i+\frac{1}{2}, j-1}\right| \Delta \varphi_{i+\frac{1}{2}, j-1}-\left|u_{i-\frac{1}{2}, j-1}\right| \Delta \varphi_{i-\frac{1}{2}, j-1}\right)\right) .
\end{aligned}
$$

In these equations the following quantities are used

$$
\begin{gathered}
\gamma_{i, j}=1-\frac{\lambda_{y}}{2}\left(v_{i, j+\frac{1}{2}}^{+}+v_{i, j-\frac{1}{2}}^{-}\right), \quad \beta_{i, j}=1-\frac{\lambda_{x}}{2}\left(u_{i+\frac{1}{2}, j}^{+}+u_{i-\frac{1}{2}, j}^{-}\right), \\
Q_{i \pm \frac{1}{2}, j}=\left|u_{i \pm \frac{1}{2}, j}\right|\left(\gamma_{i, j}-\sigma_{i \pm \frac{1}{2}, j}\right), \quad R_{i, j \pm \frac{1}{2}}=\left|v_{i, j \pm \frac{1}{2}}\right|\left(\beta_{i, j}-\nu_{i, j \pm \frac{1}{2}}\right), \\
\sigma_{i+\frac{1}{2}, j}=\max \left(\lambda_{x}\left|u_{i+\frac{1}{2}, j}\right|, \lambda_{y}\left(v_{i+1, j-\frac{1}{2}}^{+}+v_{i+1, j+\frac{1}{2}}^{+}\right), \lambda_{y}\left(v_{i, j-\frac{1}{2}}^{+}+v_{i, j+\frac{1}{2}}^{-}\right)\right), \\
\nu_{i, j+\frac{1}{2}}=\max \left(\lambda_{y}\left|v_{i, j+\frac{1}{2}}\right|, \lambda_{x}\left(u_{i-\frac{1}{2}, j+1}^{+}+u_{i+\frac{1}{2}, j+1}^{-}\right), \lambda_{x}\left(u_{i-\frac{1}{2}, j}^{+}+u_{i+\frac{1}{2}, j}^{-}\right)\right) .
\end{gathered}
$$

Unfortunately, this scheme is not $L^{\infty}$-stable yet. In order to ensure stability, a flux limiter $\phi$ defined by

$$
\phi(x)=1-\min \left(|1-x|, \frac{1}{|1-x|}\right)(1-x),
$$

is used. It fulfills the relation

$$
-2 \alpha \leq \beta \frac{\phi(r)}{r}-\alpha \phi(s) \leq 2 \beta, \quad \forall \alpha, \beta \geq 0, \forall r, s .
$$

Then, by introducing

$$
\phi_{i+\frac{1}{2}, j}^{x}=\left\{\begin{array}{ll}
\phi\left(\frac{u_{i-\frac{1}{2}, j}^{+} \Delta \varphi_{i-\frac{1}{2}, j}}{u_{i+\frac{1}{2}, j}^{+} \Delta \varphi_{i+\frac{1}{2}, j}}\right) & \text { if } u_{i+\frac{1}{2}, j} \geq 0 \\
\phi\left(\frac{u_{i+\frac{3}{2}, j}^{-} \Delta \varphi_{i+\frac{3}{2}, j}}{u_{i+\frac{1}{2}, j}^{-} \Delta \varphi_{i+\frac{1}{2}, j}}\right) & \text { if } u_{i+\frac{1}{2}, j}<0
\end{array},\right.
$$

and

$$
\phi_{i, j+\frac{1}{2}}^{y}=\left\{\begin{array}{ll}
\phi\left(\frac{v_{i, j-\frac{1}{2}}^{+} \Delta \varphi_{i, j-\frac{1}{2}}}{v_{i, j+\frac{1}{2}}^{+} \Delta \varphi_{i, j+\frac{1}{2}}}\right) & \text { if } v_{i, j+\frac{1}{2}} \geq 0 \\
\phi\left(\frac{v_{i, j+\frac{3}{2}}^{-} \Delta \varphi_{i, j+\frac{3}{2}}}{v_{i, j+\frac{1}{2}}^{-} \Delta \varphi_{i, j+\frac{1}{2}}}\right) & \text { if } v_{i, j+\frac{1}{2}}<0
\end{array},\right.
$$


in the anti-diffusive terms, (4.4) is replaced by the scheme

$$
\begin{aligned}
\varphi_{i, j}^{n+1}= & \varphi_{i, j}^{*}-\frac{\lambda_{x}}{2}\left(Q_{i+\frac{1}{2}, j} \phi_{i+\frac{1}{2}, j}^{x} \Delta \varphi_{i+\frac{1}{2}, j}-Q_{i-\frac{1}{2}, j} \phi_{i-\frac{1}{2}, j}^{x} \Delta \varphi_{i-\frac{1}{2}, j}\right) \\
& -\frac{\lambda_{y}}{2}\left(R_{i, j+\frac{1}{2}} \phi_{i, j+\frac{1}{2}}^{y} \Delta \varphi_{i, j+\frac{1}{2}}-R_{i, j-\frac{1}{2}} \phi_{i, j-\frac{1}{2}}^{y} \Delta \varphi_{i, j-\frac{1}{2}}\right) \\
& -\frac{\lambda_{x} \lambda_{y}}{4}\left(u_{i+\frac{1}{2}, j}^{-}\left(\left|v_{i+1, j+\frac{1}{2}}\right| \phi_{i+1, j+\frac{1}{2}}^{y} \Delta \varphi_{i+1, j+\frac{1}{2}}-\left|v_{i+1, j-\frac{1}{2}}\right| \phi_{i+1, j-\frac{1}{2}}^{y} \Delta \varphi_{i+1, j-\frac{1}{2}}\right)\right. \\
& +u_{i-\frac{1}{2}, j}^{+}\left(\left|v_{i-1, j+\frac{1}{2}}\right| \phi_{i-1, j+\frac{1}{2}}^{y} \Delta \varphi_{i-1, j+\frac{1}{2}}-\left|v_{i-1, j-\frac{1}{2}}\right| \phi_{i-1, j-\frac{1}{2}}^{y} \Delta \varphi_{i-1, j-\frac{1}{2}}\right) \\
& +v_{i, j+\frac{1}{2}}^{-}\left(\left|u_{i+\frac{1}{2}, j+1}\right| \phi_{i+\frac{1}{2}, j+1}^{x} \Delta \varphi_{i+\frac{1}{2}, j+1}-\left|u_{i-\frac{1}{2}, j+1}\right| \phi_{i-\frac{1}{2}, j+1}^{x} \Delta \varphi_{i-\frac{1}{2}, j+1}\right) \\
& \left.+v_{i, j-\frac{1}{2}}^{+}\left(\left|u_{i+\frac{1}{2}, j-1}\right| \phi_{i+\frac{1}{2}, j-1}^{x} \Delta \varphi_{i+\frac{1}{2}, j-1}-\left|u_{i-\frac{1}{2}, j-1}\right| \phi_{i-\frac{1}{2}, j-1}^{x} \Delta \varphi_{i-\frac{1}{2}, j-1}\right)\right) .
\end{aligned}
$$

In these conditions, the stability result proved in [25] is the following.

\section{Proposition 4.2}

The scheme defined by (4.3) and (4.5) is $L^{\infty}$-stable under the CFL condition

$$
\begin{aligned}
& \max \left(\frac{\lambda_{y}}{2}\left(v_{i, j+\frac{1}{2}}^{+}+v_{i, j-\frac{1}{2}}^{-}\right)+\sigma_{i \pm \frac{1}{2}, j}, \frac{\lambda_{x}}{2}\left(u_{i+\frac{1}{2}, j}^{+}+u_{i-\frac{1}{2}, j}^{-}\right)+\nu_{i, j \pm \frac{1}{2}},\right. \\
& 2 \lambda_{x}\left(u_{i-\frac{1}{2}, j}^{+}+u_{i+\frac{1}{2}, j}^{-}\right)\left(1-\frac{\lambda_{y}}{2}\left(\left|v_{i, j+\frac{1}{2}}\right|+\left|v_{i, j-\frac{1}{2}}\right|\right)\right) \\
& \left.+2 \lambda_{y}\left(v_{i, j-\frac{1}{2}}^{+}+v_{i, j+\frac{1}{2}}^{-}\right)\left(1-\frac{\lambda_{x}}{2}\left(\left|u_{i+\frac{1}{2}, j}\right|+\left|u_{i-\frac{1}{2}, j}\right|\right)\right)\right) \leq 1
\end{aligned}
$$

In the following section, the discretization of the inertial term $\mathbf{v} . \nabla \mathbf{v}$ in the Navier-Stokes equation is performed by using a straightforward upwind scheme. This implies a stability condition which is exactly (4.1). Hence, whatever the scheme chosen for the transport equation, the time step $\delta t$ must satisfy (4.1). In order to avoid a too high numerical diffusion in the Navier-Stokes equation, the time step is taken in all cases

$$
\delta t=\min \left(\frac{0.9}{\frac{1}{\delta x} \sup _{i, j}\left|u_{i-\frac{1}{2}, j}\right|+\frac{1}{\delta y} \sup _{i, j}\left|v_{i, j-\frac{1}{2}}\right|}, \delta t_{\text {max }}\right),
$$

where $\delta t_{\max }$ is chosen to ensure a sufficient accuracy, and the coefficient 0.9 to avoid the limit cases of stability.

This choice of the time step is unfortunately not able to ensure (4.6) if the Rasetarinera scheme is used because (4.6) turns out to be more restrictive than (4.1) for $\delta t$. To overcome this difficulty, $K$ being the smallest integer such that $\delta t^{\prime}=\delta t / K$ satisfies (4.6), the scheme (4.3)-(4.5) is performed $K$ times, with a time step $\delta t^{\prime}$ to obtain the solution at the time $t+\delta t$. In the computations presented in section 5 , the integer $K$ satisfies $1 \leq K \leq 4$, so that this method does not induce a too high additional cost of computational time.

\subsubsection{Navier-Stokes equations}

At each Navier-Stokes step of the time discretization, several Stokes problems have to be solved with the augmented Lagrangian method. This leads to the resolution of elliptic systems of the form

$$
\tilde{\rho} \mathbf{v}-\omega_{1} \operatorname{div}(2 \tilde{\eta} \mathbf{D}(\mathbf{v}))-\omega_{2} \nabla \operatorname{div} \mathbf{v}=\left(\begin{array}{c}
a \\
b
\end{array}\right)
$$

where $\tilde{\rho}=\rho\left(\varphi^{n+1}\right), \tilde{\eta}=\eta\left(\varphi^{n+1}\right)$ are known at the points $A_{i, j}$, and $a, b$ are respectively known at the points $A_{i-\frac{1}{2}, j}$ and $A_{i, j-\frac{1}{2}}$. This equation can be written in the two coordinates $u$ and $v$ as

$$
\begin{aligned}
& \tilde{\rho} u-\omega_{1} \frac{\partial}{\partial x}\left(2 \tilde{\eta} \frac{\partial u}{\partial x}\right)-\omega_{1} \frac{\partial}{\partial y}\left(\tilde{\eta}\left(\frac{\partial u}{\partial y}+\frac{\partial v}{\partial x}\right)\right)-\omega_{2} \frac{\partial}{\partial x}\left(\frac{\partial u}{\partial x}+\frac{\partial v}{\partial y}\right)=a \\
& \tilde{\rho} v-\omega_{1} \frac{\partial}{\partial x}\left(\tilde{\eta}\left(\frac{\partial u}{\partial y}+\frac{\partial v}{\partial x}\right)\right)-\omega_{1} \frac{\partial}{\partial y}\left(2 \tilde{\eta} \frac{\partial v}{\partial y}\right)-\omega_{2} \frac{\partial}{\partial y}\left(\frac{\partial u}{\partial x}+\frac{\partial v}{\partial y}\right)=b .
\end{aligned}
$$


Now, (4.9)-(4.10) are discretized using the classical second order finite differences scheme for elliptic equations. At the point $A_{i-\frac{1}{2}, j}$, equation (4.9) becomes

$$
\begin{aligned}
\tilde{\rho}_{i-\frac{1}{2}, j} u_{i-\frac{1}{2}, j}- & \frac{\omega_{1}}{\delta x}\left\{2 \tilde{\eta}_{i, j} \frac{u_{i+\frac{1}{2}, j}-u_{i-\frac{1}{2}, j}}{\delta x}-2 \tilde{\eta}_{i-1, j} \frac{u_{i-\frac{1}{2}, j}-u_{i-\frac{3}{2}, j}}{\delta x}\right\} \\
- & \frac{\omega_{1}}{\delta y}\left\{\tilde{\eta}_{i-\frac{1}{2}, j+\frac{1}{2}}\left(\frac{u_{i-\frac{1}{2}, j+1}-u_{i-\frac{1}{2}, j}}{\delta y}+\frac{v_{i, j+\frac{1}{2}}-v_{i-1, j+\frac{1}{2}}}{\delta x}\right)\right. \\
& \left.-\tilde{\eta}_{i-\frac{1}{2}, j-\frac{1}{2}}\left(\frac{u_{i-\frac{1}{2}, j}-u_{i-\frac{1}{2}, j-1}}{\delta y}+\frac{v_{i, j-\frac{1}{2}}-v_{i-1, j-\frac{1}{2}}}{\delta x}\right)\right\} \\
- & \frac{\omega_{2}}{\delta x}\left\{\left(\frac{u_{i+\frac{1}{2}, j}-u_{i-\frac{1}{2}, j}}{\delta x}+\frac{v_{i, j+\frac{1}{2}}-v_{i, j-\frac{1}{2}}}{\delta y}\right)\right. \\
& \left.-\left(\frac{u_{i-\frac{1}{2}, j}-u_{i-\frac{3}{2}, j}}{\delta x}+\frac{v_{i-1, j+\frac{1}{2}}-v_{i-1, j-\frac{1}{2}}}{\delta y}\right)\right\} \\
= & a_{i-\frac{1}{2}, j} .
\end{aligned}
$$

At the point $A_{i, j-\frac{1}{2}}$, equation (4.10) reads, in its discrete form,

$$
\begin{aligned}
\tilde{\rho}_{i, j-\frac{1}{2}} v_{i, j-\frac{1}{2}}-\frac{\omega_{1}}{\delta x} & \left\{\tilde{\eta}_{i+\frac{1}{2}, j-\frac{1}{2}}\left(\frac{u_{i+\frac{1}{2}, j}-u_{i+\frac{1}{2}, j-1}}{\delta y}+\frac{v_{i+1, j-\frac{1}{2}}-v_{i, j-\frac{1}{2}}}{\delta x}\right)\right. \\
& \left.-\tilde{\eta}_{i-\frac{1}{2}, j-\frac{1}{2}}\left(\frac{u_{i-\frac{1}{2}, j}-u_{i-\frac{1}{2}, j-1}}{\delta y}+\frac{v_{i, j-\frac{1}{2}}-v_{i-1, j-\frac{1}{2}}}{\delta x}\right)\right\} \\
- & \frac{\omega_{1}}{\delta y}\left\{2 \tilde{\eta}_{i, j} \frac{v_{i, j+\frac{1}{2}}-v_{i, j-\frac{1}{2}}}{\delta y}-2 \tilde{\eta}_{i, j-1} \frac{v_{i, j-\frac{1}{2}}-v_{i, j-\frac{3}{2}}}{\delta y}\right\} \\
- & \frac{\omega_{2}}{\delta y}\left\{\left(\frac{u_{i+\frac{1}{2}, j}-u_{i-\frac{1}{2}, j}}{\delta x}+\frac{v_{i, j+\frac{1}{2}}-v_{i, j-\frac{1}{2}}}{\delta y}\right)\right. \\
& \left.-\left(\frac{u_{i+\frac{1}{2}, j-1}-u_{i-\frac{1}{2}, j-1}}{\delta x}+\frac{v_{i, j-\frac{1}{2}}-v_{i, j-\frac{3}{2}}}{\delta y}\right)\right\} \\
= & b_{i, j-\frac{1}{2}} .
\end{aligned}
$$

The values of $\tilde{\eta}_{i \pm \frac{1}{2}, j \pm \frac{1}{2}}$ (resp. $\tilde{\rho}_{i-\frac{1}{2}, j}, \tilde{\rho}_{i, j-\frac{1}{2}}$ ) are obtained from the values of $\tilde{\eta}$ (resp. $\tilde{\rho}$ ) at the points $A_{i, j}$ by interpolation using arithmetic or harmonic mean (this last one is supposed to give better results).

To obtain the right-hand side $a$ and $b$ in the equation (4.8), we put (3.9) in the form (4.8) to get, after dropping the time discretization superscript

$$
\begin{aligned}
& \tilde{\rho} u-\delta t\left(\frac{\partial p}{\partial x}-\tilde{\rho} \mathbf{v} \cdot \nabla u+\mathcal{K} \mu \frac{\partial \varphi}{\partial x}+\varepsilon \mathcal{K} \frac{1-\varphi^{2}}{4} \frac{\partial}{\partial x}\left(\frac{\mu}{\tilde{\rho}}\right)+\rho g^{x}\right)=a, \\
& \tilde{\rho} v-\delta t\left(\frac{\partial p}{\partial y}-\tilde{\rho} \mathbf{v} \cdot \nabla v+\mathcal{K} \mu \frac{\partial \varphi}{\partial y}+\varepsilon \mathcal{K} \frac{1-\varphi^{2}}{4} \frac{\partial}{\partial y}\left(\frac{\mu}{\tilde{\rho}}\right)+\rho g^{y}\right)=b .
\end{aligned}
$$

These expressions are then discretized in a straightforward way

$$
\begin{aligned}
a_{i-\frac{1}{2}, j}=\tilde{\rho}_{i-\frac{1}{2}, j} u_{i-\frac{1}{2}, j}-\delta t & \left(\frac{p_{i, j}-p_{i-1, j}}{\delta x}-\tilde{\rho}_{i-\frac{1}{2}, j}(\mathbf{v} . \nabla u)_{i-\frac{1}{2}, j}+\mathcal{K} \frac{\mu_{i-1, j}+\mu_{i, j}}{2} \frac{\varphi_{i, j}-\varphi_{i-1, j}}{\delta x}\right. \\
& \left.\frac{\varepsilon \mathcal{K}}{4 \delta x}\left\{1-\left(\frac{\varphi_{i, j}+\varphi_{i-1, j}}{2}\right)^{2}\right\}\left\{\frac{\mu_{i, j}}{\tilde{\rho}_{i, j}}-\frac{\mu_{i-1, j}}{\tilde{\rho}_{i-1, j}}\right\}+\tilde{\rho}_{i-\frac{1}{2}, j} g_{i-\frac{1}{2}, j}^{x}\right),
\end{aligned}
$$




$$
\begin{aligned}
b_{i, j-\frac{1}{2}}=\tilde{\rho}_{i, j-\frac{1}{2}} v_{i, j-\frac{1}{2}}-\delta t & \left(\frac{p_{i, j}-p_{i, j-1}}{\delta y}-\tilde{\rho}_{i, j-\frac{1}{2}}(\mathbf{v} \cdot \nabla v)_{i, j-\frac{1}{2}}+\mathcal{K} \frac{\mu_{i, j-1}+\mu_{i, j}}{2} \frac{\varphi_{i, j}-\varphi_{i, j-1}}{\delta y}\right. \\
& \left.\frac{\varepsilon \mathcal{K}}{4 \delta y}\left\{1-\left(\frac{\varphi_{i, j}+\varphi_{i, j-1}}{2}\right)^{2}\right\}\left\{\frac{\mu_{i, j}}{\tilde{\rho}_{i, j}}-\frac{\mu_{i, j-1}}{\tilde{\rho}_{i, j-1}}\right\}+\tilde{\rho}_{i, j-\frac{1}{2}} g_{i, j-\frac{1}{2}}^{y}\right)
\end{aligned}
$$

where the terms $(\mathbf{v} \cdot \nabla u)_{i-\frac{1}{2}, j}$ and $(\mathbf{v} \cdot \nabla v)_{i, j-\frac{1}{2}}$ are computed using a classical upwind scheme. Note that these terms need to linearly interpolate the values of $u$ and $v$, and that the stability condition imposed is exactly (4.1) which implies the choice (4.7) for the time step.

Finally, a sparse non-symmetric linear system in the unknowns $\left(u_{i-\frac{1}{2}, j}\right),\left(v_{i, j-\frac{1}{2}}\right)$ has to be solved.

\subsection{Comparisons between Murman and Rasetarinera schemes}

It is shown here the importance of the Rasetarinera scheme in order to limit the numerical diffusion.
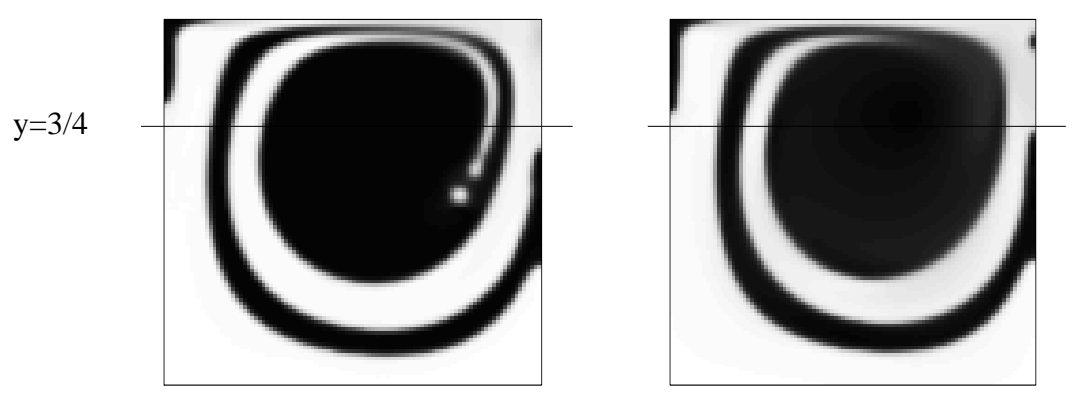

Figure 2: Rasetarinera scheme (left-hand side) and Murman scheme (right-hand side)

Figure 2 shows the computations at the same time of a driven cavity with the same initial data and the same parameters, the first one being computed with the Murman scheme and the second one with the Rasetarinera scheme.

One can clearly see the importance of the numerical diffusion for the Murman scheme. For example, the one obtained with the Rasetarinera scheme presents a breakup in the pattern which is not the case with the Murman scheme.

Figure 3 shows more precisely the difference of the two solutions along the line $y=3 / 4$ : the interfaces, as well as the pure fluid areas, are more accurately computed with the Rasetarinera scheme. Furthermore, this scheme is almost of the second order and so a higher accuracy is reached in the computations using this more complex scheme. 


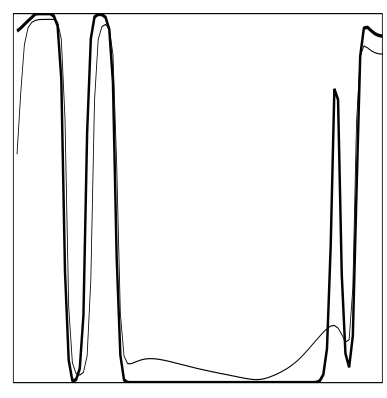

- Murman _ Rasetarinera

Figure 3: Plot of $\varphi(., 3 / 4)$ for the two schemes (see figure 2)

\section{$5 \quad$ Numerical results and validation}

In the computations presented here, the following values of the parameters were used :

$$
\alpha^{2}=5.10^{-5}, \quad B(\varphi)=1-\varphi^{2}, \quad F(\varphi)=\varphi^{4} / 4-\varphi^{2} / 2,
$$

the discretization grid used is $100 \times 100$, so that the interface width is of the same order as $\delta x$ and $\delta y$.

As shown in the previous section, the Rasetarinera scheme gives more accurate results than the Murman one. For this reason, all the results presented below are obtained using this scheme. It is shown in this section that the model is able to take into account several physical situations in which diphasic fluids are involved. Moreover, in the sequel, the role played by the different terms and coefficients in the equations is pointed out.

Remark : One can check that the scheme is conservative in the sense that the mean of $\varphi$ is conserved along the time, as in the continuous equation (2.21). Another important property of the Cahn-Hilliard equation is that, under suitable assumptions on the data, on $B$ and $F$ (for example when $B$ is degenerate [5], or when $F$ has a logarithmic form), the order parameter $\varphi$ takes always values in the physical-meaningful interval $[-1,1]$. In the following computations, this property is conserved (at the order 2).

\subsection{Regularized driven cavity}

In this first test, we suppose that the fluids have the same density and viscosity, so that the following results can be compared to the ones in [10].

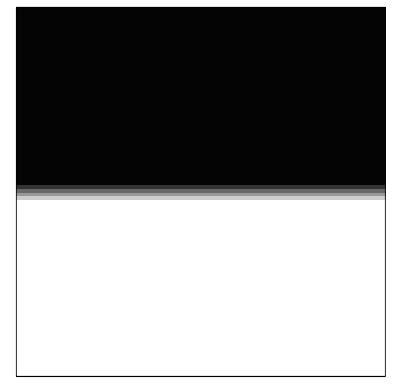

Figure 4: Initial condition for the driven cavity

At the upper boundary of the square cavity $[0,1] \times[0,1]$, the velocity is imposed to be

$$
u=C x(1-x), \quad v=0,
$$


where $C$ is a constant, whereas $\mathbf{v}=0$ is imposed on the remaining part of the boundary. The unique reason why the imposed velocity is regularized is to be able to compare the results with the ones obtained in [10]. The initial condition chosen is the configuration of $\varphi$ given by figure 4 at rest.

- Effects of capillarity : In order to find out the influence on the solution of the capillary forces, some tests are performed with $\mathcal{R} e=500$ and $P e=1000$ for different values of the capillary number $\mathcal{K}$.

$$
\mathcal{K}=0
$$
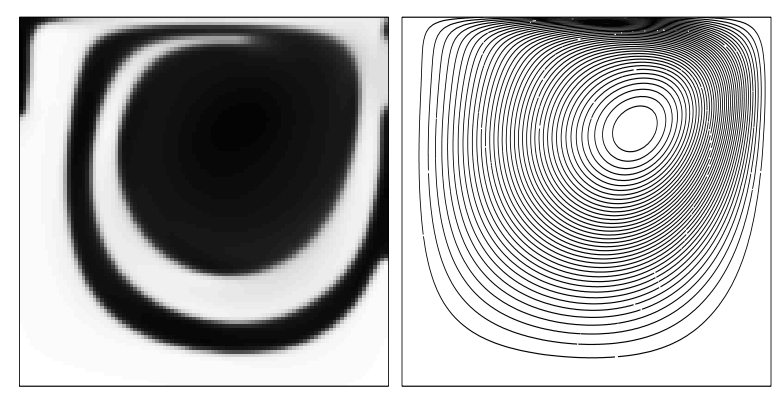

$$
\mathcal{K}=\frac{10}{\mathcal{R} e}
$$
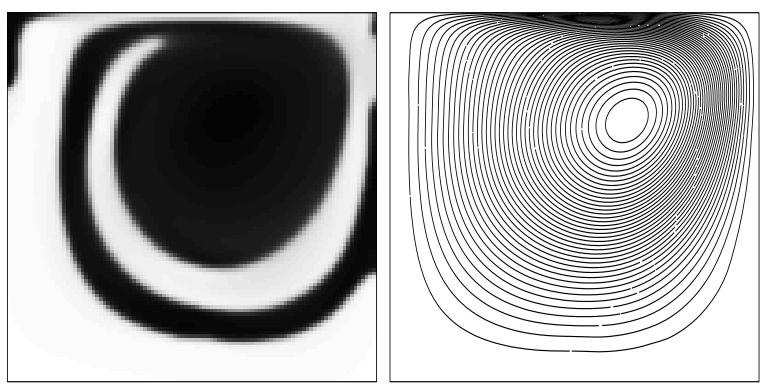

$$
\mathcal{K}=\frac{0.1}{\mathcal{R} e}
$$
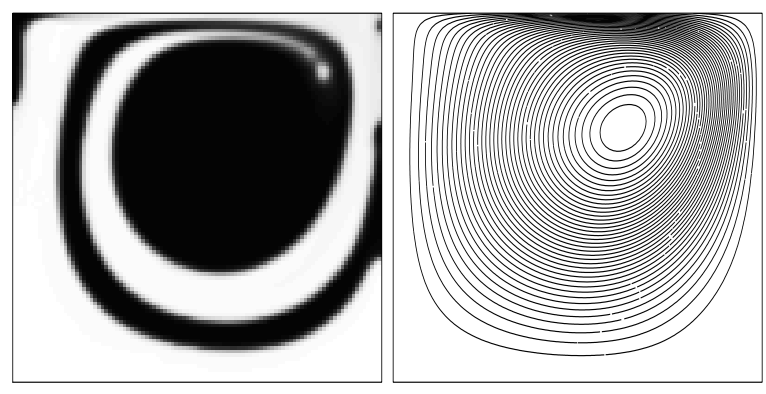

$$
\mathcal{K}=\frac{100}{\mathcal{R} e}
$$
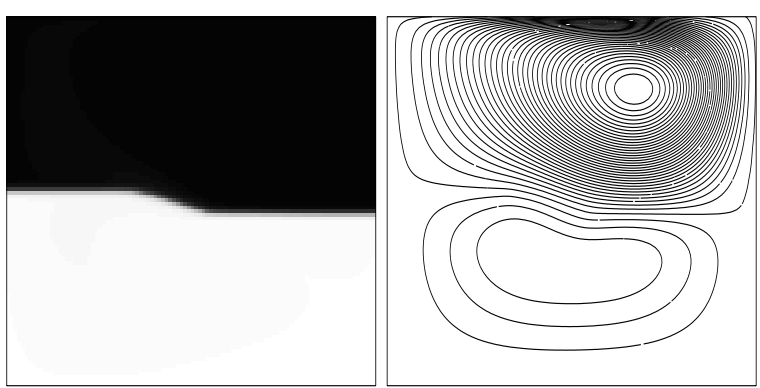

Figure 5: Influence of the capillarity

One can see that when the the viscous forces are are preponderant in front of the capillary forces $\left(\mathcal{K}=\frac{0.1}{\mathcal{R e}}\right)$, the presence of capillary forces increases the effect of stretching of the interface by a flatenning process (in comparison with the case $\mathcal{K}=0$ of a passive interface). On the contrary, when the capillary forces are too important in front of the viscous forces $\left(\mathcal{K}=\frac{10}{\mathcal{R} e}\right)$ they counteract the stretching effect of the flow, as they lead to an increasing rigidity of the interface. In the case $\mathcal{K}=\frac{100}{R e}$, the viscous forces are not sufficient in front of capillarity to melt the two phases and the interface remains essentially flat.

Such a study of the influence of capillary forces, is also proposed in the test case of the Rayleigh-Taylor instability in section 5.3 .

\section{- Effects of the diffusion/anti-diffusion in the Cahn-Hilliard equation :}

It is shown here that the results obtained through the complete model including the Cahn-Hilliard term accounts numerically for the physical interfacial thickness. More precisely, even if a very anti-diffusive scheme is used for the transport, one might expect to observe significant numerical diffusion at the interface at large times. In fact, such a behavior is not observed, the reason being that the Cahn-Hilliard term contains a physical-relevant anti-diffusive part due to the non-convexity of the Cahn-Hilliard potential $F$, which ensures a local equilibrium between the two phases with a constant thickness of the interface. 
In order to underline these facts, the results for the complete model are compared to the results obtained with $\mathcal{P} e=+\infty$, a limit model with only a pure interfacial transport (nonhomogeneous incompressible fluids model [20], [27]). The two results presented are taken at the same computational time, and the physical parameters are in both cases : $\mathcal{R} e=500$ and $\mathcal{K}=2 \cdot 10^{-4}$.

$$
\mathcal{P} e=1000
$$

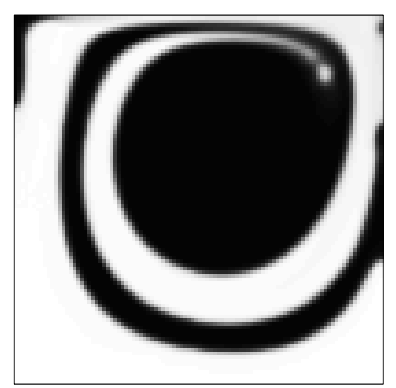

$$
\mathcal{P} e=+\infty
$$

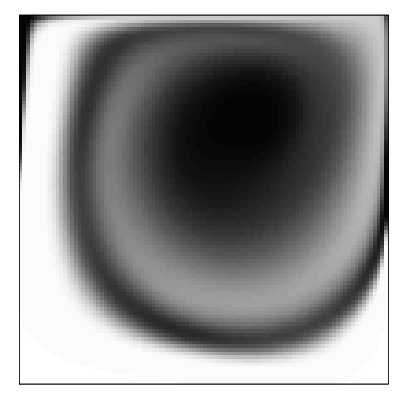

Figure 6: Influence of the Peclet number

It is now clear that with the model introduced here, it is very easy to follow the interface from a numerical point of view, compared to the nonhomogeneous fluid model. Moreover, the results presented here were performed without any artificial modification of the model. This is not the case for the level set methods [28] for example, in which a regularization of the Heavyside and Dirac functions is needed and a renormalization process is necessary at each time step. This leads to the introduction of a numerical interface thickness whereas the model is theoretically concerned with interfaces without thickness.

Remark also that with the complete model $(\mathcal{P} e<+\infty)$, the interface is allowed to move along the boundaries by diffusion, even if the velocity vanishes at the boundary.

\subsection{Spinodal decomposition and pattern formation in a channel under shear}

In this paragraph the case of a channel under shear with two fluids of same density is considered. The initial condition is chosen randomly around 0 , and is the same for all the computations presented here. The parameters used are $\mathcal{K}=1 / 500, \mathcal{R} e=500$ and $\mathcal{P} e=1000$. The results obtained are presented simultaneously for a shear velocity equal to 1 and for a shear velocity 10 at equivalent times (the time scales are not the same).

In the two cases, the evolution to the stationary solution can be divided into two successive stages. In the first one, one can see the formation of patterns in the mixture under the influence of the Cahn-Hilliard term (this phenomenon is know as the spinodal decomposition). Then in the second stage, one can see the influence of the shear velocity which tends to organize the patterns in some parallel layers of almost pure fluids.

Moreover, the patterns formed in the early stage are quite different when the shear velocity increases, and the number of layers in the late stage increases when the shear is higher. This behavior is exactly the one observed by the physicists in their experiments ([11], [23]). 
Computers and Fluids 31 numéro 1, pp 41-68 (2002)

$$
\text { Shear velocity }=1 \quad \text { Shear velocity }=10
$$
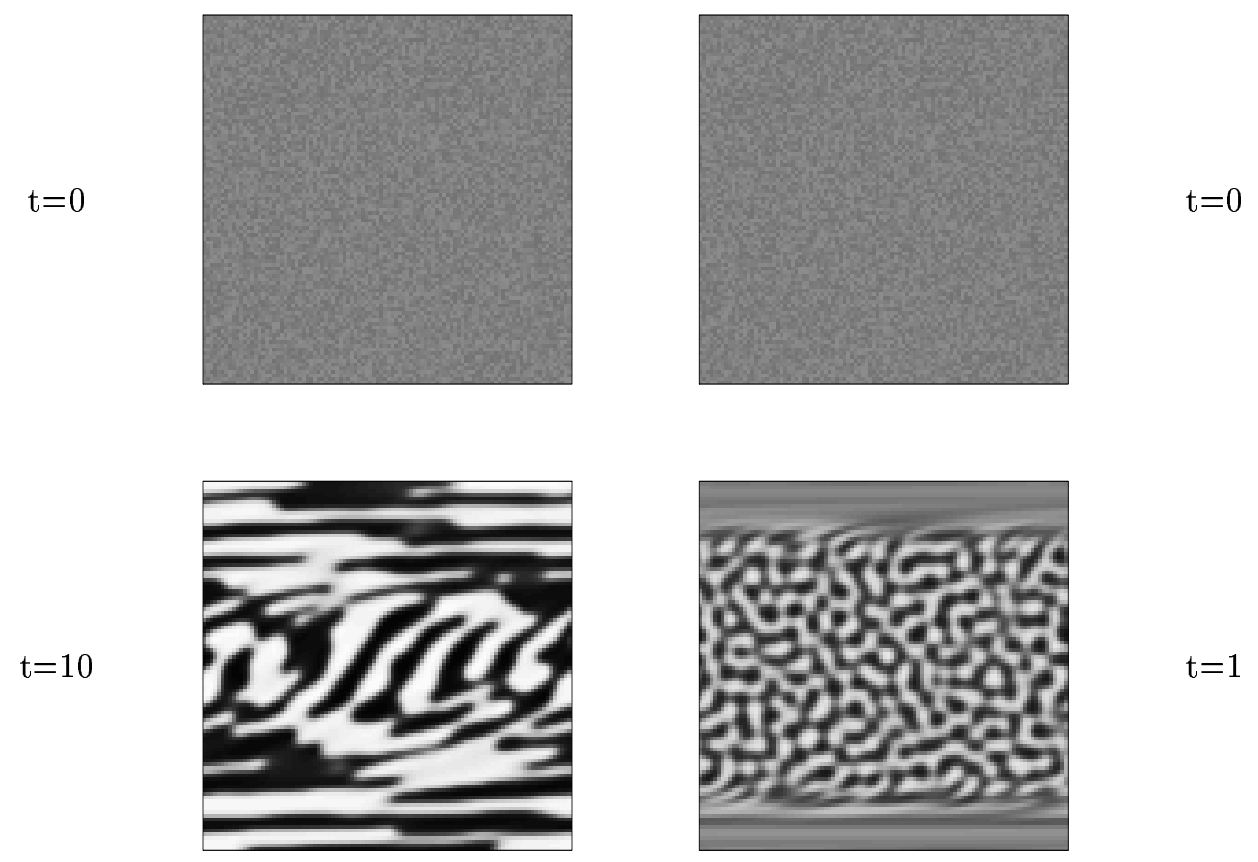

$\mathrm{t}=1$
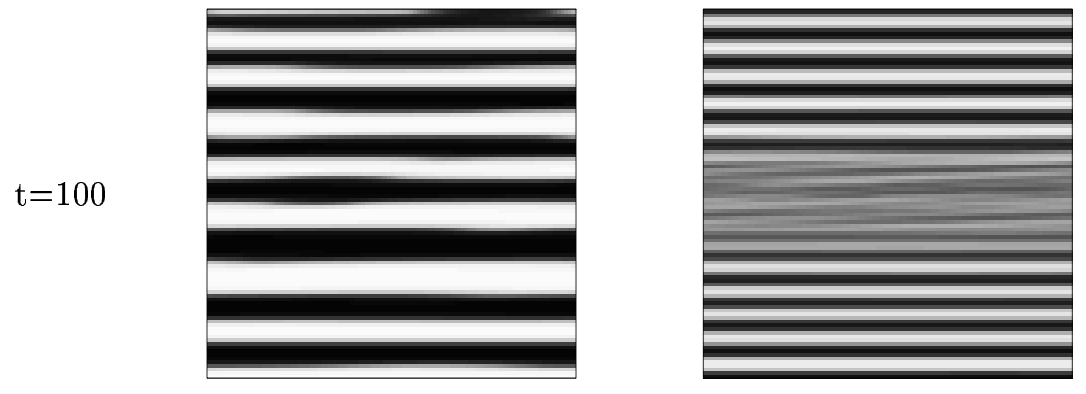

$\mathrm{t}=30$

Figure 7: Spinodal decomposition

\subsection{Rayleigh-Taylor instability}

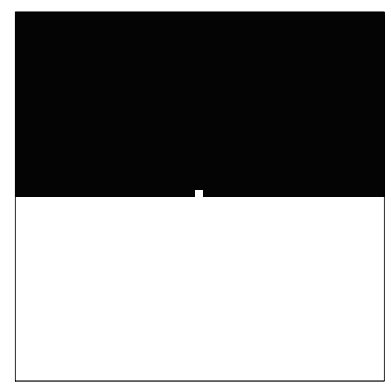

Figure 8: initial condition for the Rayleigh-Taylor instability 
This test case is another example with Dirichlet boundary conditions. The instability is created when one put a heavy fluid upon a lighter one and when the equilibrium state is perturbated. The density contrast between the two fluids chosen here is

$$
\rho_{1}^{0} / \rho_{2}^{0}=1000,
$$

and the viscosities are supposed to be equal. The computations presented are performed with the same initial condition for $\varphi$ (see figure 8 ) at rest $(\mathbf{v}=0$ at $t=0)$.

The various behaviors of the system, when the capillary number varies, are shown at equivalent times. It is known that the capillarity is directly linked with the surface tension (energy excess at the interface) and so with the curvature of the interface (see [10], [18]). This fact is effectively observed here: the capillary forces seem to make the interface as flat as possible.

Moreover, when the capillarity is really important $\left(\mathcal{K}=\frac{10}{\mathcal{R} e}\right)$, the capillary forces are preponderant at the very beginning of the process, when the system has no inertia. Hence, before the instability begins to develop, the surface tension causes the flattening of the interface. That's the reason why in this case, the system evolves in an opposite way compared to the other cases.

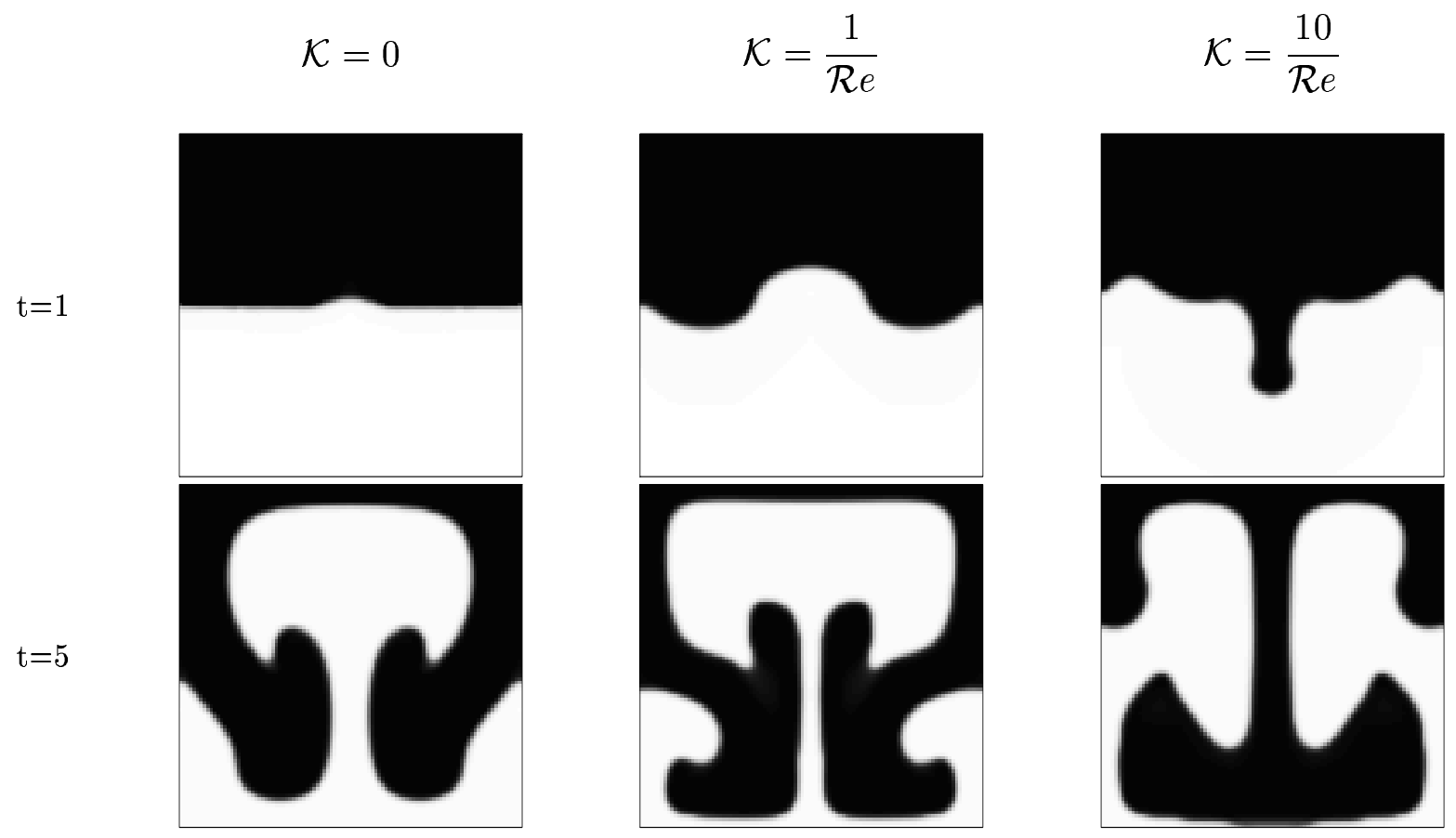

Figure 9: Rayleigh-Taylor instability (first part) 
Computers and Fluids 31 numéro 1, pp 41-68 (2002)
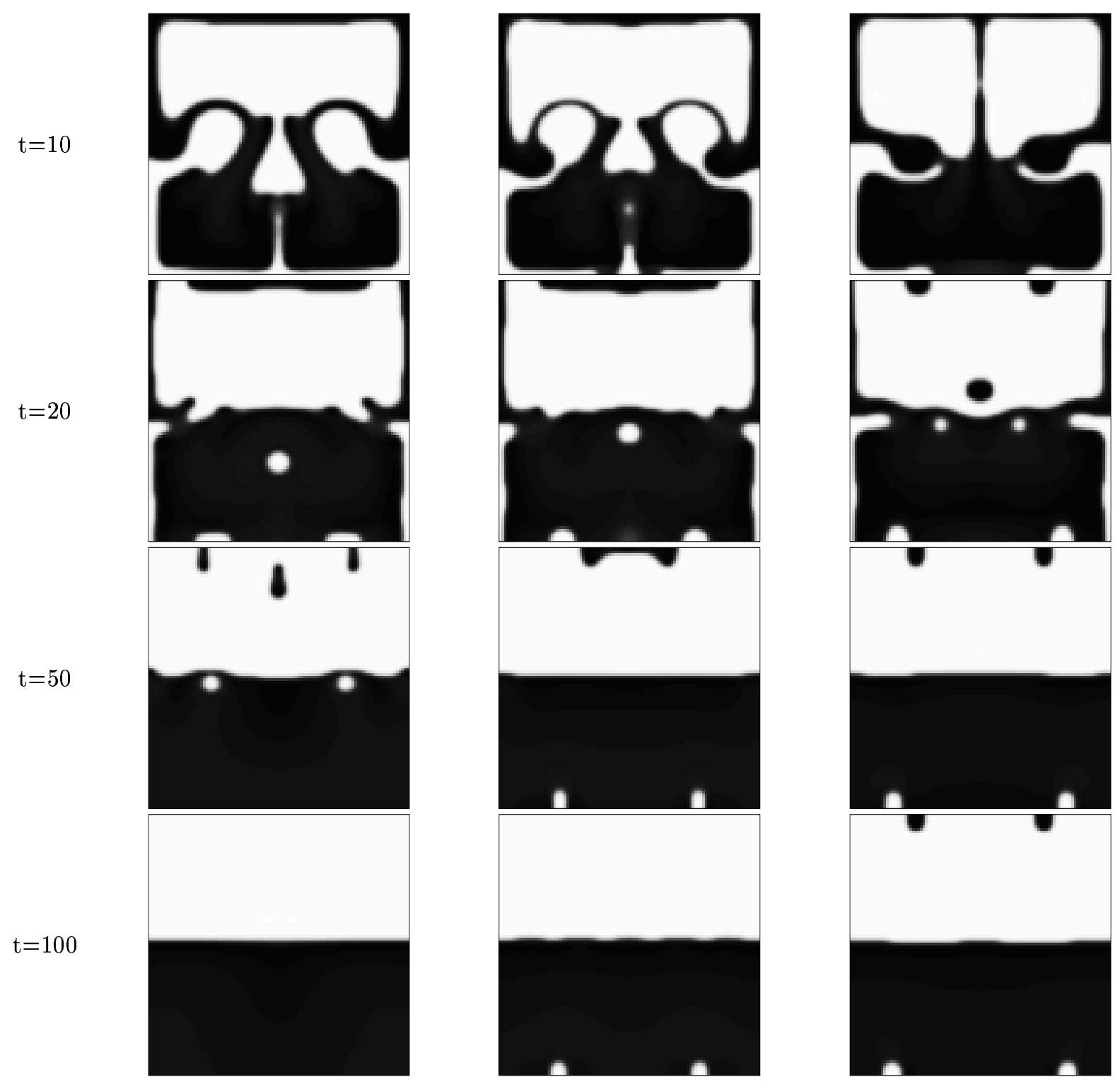

Figure 9: Rayleigh-Taylor instability (second part) 


\subsection{Falling droplet}

To point out the role played by the viscosity contrast of the two fluids, and the way the model is able to take this contrast into account, two tests are presented here. The parameters considered are $\mathcal{P} e=1000$ and $\mathcal{K}=0.01$, the density contrast being the same than in the case of the Rayleigh-Taylor instability

$$
\rho_{1}^{0} / \rho_{2}^{0}=1000 .
$$

In a first computation (figure 10), the case of a droplet of water in the air is simulated with a variable viscosity function $\eta(\varphi)$ such that the droplet is 50 times as viscous as the other fluid. The Reynolds number in the air is chosen to be 1000 .

In the second one (figure 11), the two fluids are supposed to have the same viscosities $(\eta(\varphi) \equiv 1)$ and we take $\mathcal{R} e=1000$. One may remember that, for example, mercury and water can be considered in this situation (same viscosities, but different densities).
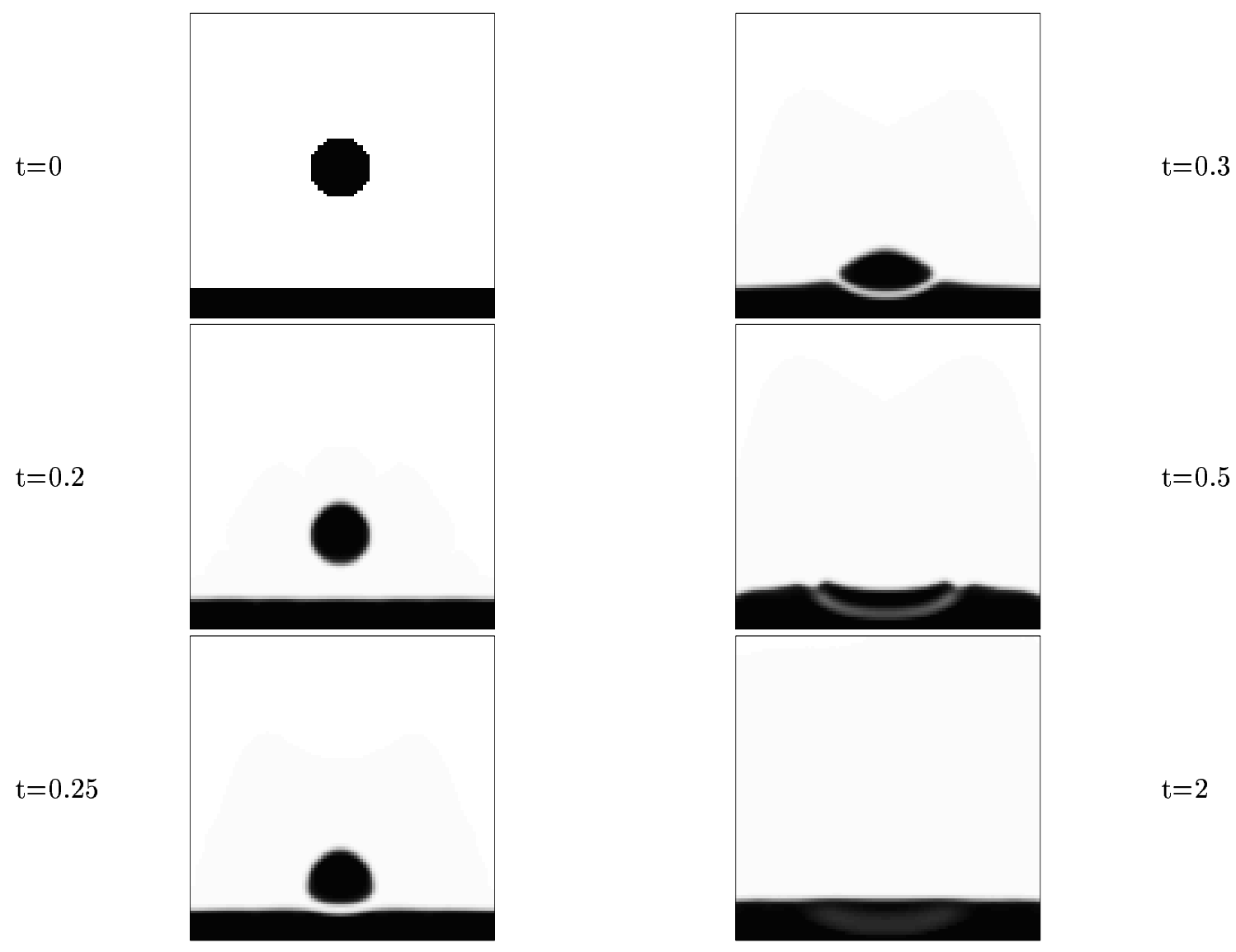

Figure 10: falling droplet : test 1 
Computers and Fluids 31 numéro 1, pp 41-68 (2002)

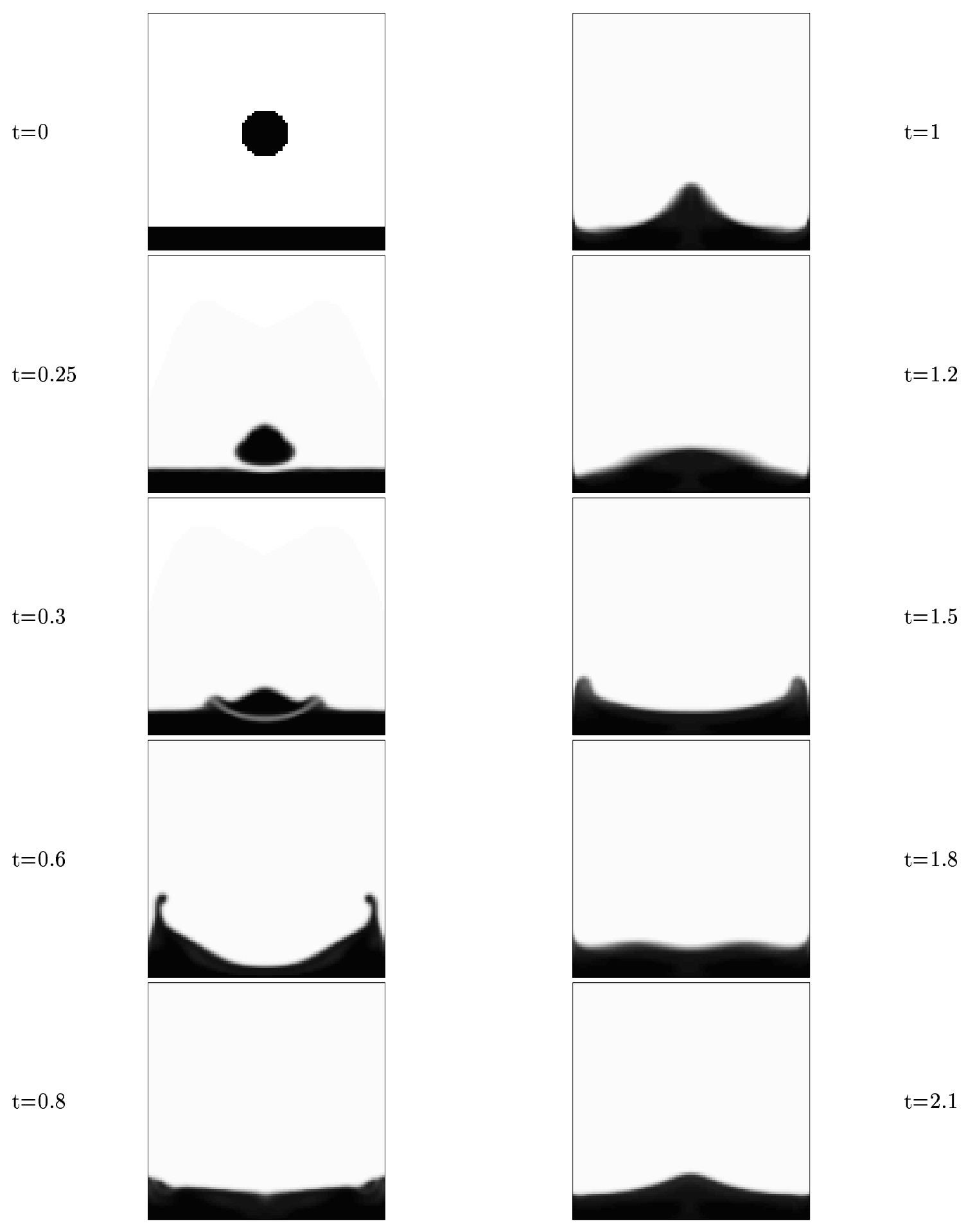

Figure 11: falling droplet : test 2 
In the first test (figure 10), no splashing occurs, whereas in the second one (figure 11), one can see between the times $t=0.6$ and $t=2.1$, that some kind of "waves" propagate horizontally in the domain alternatively from the center to the boundaries and from the boundaries to the center. Hence, the fact that the viscosities of the two fluids are different has a great influence on the evolution of the system. In fact, if the height of the domain were higher, it is clear that the splashing would also occur in the first case.

The qualitative results obtained are in agreement with the same kind of results found in the literature, in particular the shape we get at $t=0.6$ in the second test (figure 11) is very similar to the one obtained with an adaptative level set method in [28].

\section{Conclusions}

In this work, a new model for the study of incompressible mixture flows with different densities was carefully derived. This model accounts very well for the interaction between the two phases at the interface (including capillary forces), as well as for the various hydrodynamic effects. The equations obtained are basically a coupling between a Cahn-Hilliard equation and an incompressible nonhomogeneous Navier-Stokes equation.

A quite simple finite-difference discretization of the system was presented, in which it was particularly pointed out the importance of a careful treatment of the convection term. In this direction, the use of a second order anti-diffusive scheme from P. Rasetarinera was proposed and was shown to be effectively efficient. Moreover, it was shown that the model of the nonhomogeneous fluids, that is with a pure interfacial transport, is much more sensitive to the numerical diffusion appearing in the discretization of the transport term.

The simulations of spinodal decomposition under shear, which is at the origin of this study, were presented, in accordance with the physical experiments snapshots found in [23]. The other numerical results presented illustrate the wide range of physical situations which can be simulated through this model.

Acknowledgments: The author is very grateful to the professors A. Colin, P. Fabrie, D. Jamet, P. Panizza and M. Quintard for many helpful discussions and advices.

\section{References}

[1] N. Alikakos, P. Bates, G. Fusco, Slow motion for the Cahn-Hilliard equation in one space dimension, J. Diff. Eq. 90, pp 81-135 (1991).

[2] D. M. Anderson, G. B. McFadden, A. A. Wheeler, Diffuse-interface methods in fluid mechanics, Annu. Rev. Fluid Mech. Vol. 30 pp139-165 (1998).

[3] H. W. Barrett, J. F. Blowey, Finite elements approximation of the Cahn-Hilliard equation with concentration dependent mobility, Mathematics of Computation 68 No. 226, pp 487-517 (1999).

[4] P. W. Bates, P. C. Fife, The dynamics of nucleation for the Cahn-Hilliard equation, SIAM J. Appl. Math. 53 No. 4, pp 990-1008 (1993).

[5] F. Boyer, Mathematical study of multiphase flow under shear through order parameter formulation, Asymptotic Analysis 20 No. 2, pp 175-212 (1999).

[6] F. Boyer, Nonhomogeneous Cahn-Hilliard fluids, to appear in Annales de l'IHP : Analyse non linéaire (2000).

[7] C.H. Bruneau, P. Fabrie, P. Rasetarinera, An accurate finite difference scheme for solving convectiondominated diffusion equations, Internat. J. Numer. Methods Fluids 24 No. 2, pp 169-183 (1997)

[8] C.H. Bruneau, P. Rasetarinera, A finite volume method with effecicient limiters for solving conservation laws, Computational Fluid Dynamics Journal 6 No. 1, pp 23-38 (1997). 
[9] J. Carr, M. Gurtin, M. Slemrod, Structured phase transitions on a finite interval, Arch. Rational Mech. Anal. 86, pp. 317-351 (1984).

[10] R. Chella, J. Vinals, Mixing of a two-phase fluid by a cavity flow, Physical Review E 53, 3832 (1996).

[11] A. Colin, P. Panizza, Private communications.

[12] A. Debussche, L. Dettori, On the Cahn-Hilliard equation with a logarithmic free energy, Nonlinear Analysis 24, no 10, pp 1491-1514, (1995).

[13] M. Doi, Dynamics of domains and textures, Theoretical Challenges in the Dynamics of Complex Fluids, pp. 293-314, (1997).

[14] C.M. Elliott, H. Garcke, On the Cahn-Hilliard equation with degenerate mobility, Siam J. Math. Anal. 27 No. 2, pp 404-423 (1996)

[15] M. Fortin, R. Glowinski, Augmented lagrangian methods : applications to the numerical solution of boundary-value problems, Studies in Mathematics and its Applications 15, North-Holland Publishing Comp. (1983).

[16] J. D. Gunton, M. San Miguel, P.S. Sahni, in Phase transitions and critical phenomena Vol. 8, ed. by Domb and Lebowitz (Academic, London) 1983.

[17] M. E. Gurtin, D. Polignone, J. Vinals Two-phase binary fluids and immiscible fluids described by an order parameter Mathematical Models and Methods in Applied Sciences 6, 815 (1996).

[18] D. Jacqmin, Calculation of two-phase Navier-Stokes flows using phase-field modeling, Journal of Computational Physics 155, pp 96-127 (1999).

[19] D. Jasnow, J. Vinals Coarse-grained description of thermo-capillary flow Phys. Fluids, 8, 660 (1996).

[20] P.L. Lions, Mathematical Topics in Fluid Mechanics : Vol 1 incompressible models, Oxford Science Publications (1996).

[21] B. T. Nadiga, S. Zaleski, Investigations of a two-phase fluid model, Eur. J. Mech. B/Fluids, Vol. 15 no 6 , pp 885-896 (1996).

[22] T. Ohta, H. Nozaki, M. Doi, Computer simulations of domain growth under steady shear flow, J. Chem. Phys. Vol. 93, no. 4, pp 2664-2675 (1990).

[23] A. Onuki, Phase transitions of fluids in shear flow, J. Phys.: Condens. Matter 9, pp 6119-6157 (1997).

[24] K.R. Rajagopal, L. Tao, Mechanics of mixtures, Series on Advances in Mathematics for Applied Sciences, Vol. 35, World Scientific.

[25] P. Rasetarinera, Etude mathématique et numérique de la restauration biologique en milieu poreux, $\mathrm{Ph} . \mathrm{D}$. Thesis, MAB, Univ. Bordeaux 1. (1995)

[26] Y. Saad, Iterative methods for sparse linear systems, PWS-KENT Publishing company, 1996.

[27] J. Simon, Nonhomogeneous viscous incompressible fluids: existence of velocity, density, and pressure, SIAM J. Math. Anal., Vol. 21 no 5 pp 1093-1117 (1990).

[28] M. Sussman, A. S. Almgren, J. B. Bell, P. Colella, L. H. Howell, M. L. Welcome, An adaptative level set approach for incompressible two-phase flows, Journal of Computational Physics Vol. 148, pp 81-124 (1999).

[29] R. Temam, Infinite-dimensional dynamical systems in mechanics and physics, Applied Mathematial Science 68, Springer-Verlag (1997). 